\title{
Solvability of a moving contact-line problem with interface formation for an incompressible viscous fluid
}

\section{Yoshiaki Kusaka ${ }^{1 *}$}

\section{"Correspondence:}

kusaka@eng.tamagawa.ac.jp

'Department of Mathematics,

Tamagawa University, Tokyo, Japan

\begin{abstract}
We investigate the free-boundary problem of a steadily advancing meniscus in a circular capillary tube. The problem is described using the "interface formation model," which was originally introduced with the aim of avoiding the singularities that arise when classical hydrodynamics is applied to problems with a moving contact line. We prove the existence of an axially symmetric solution in weighted Hölder spaces for low meniscus speeds.
\end{abstract}

Keywords: Navier-Stokes equations; Moving contact-line problem; Incompressible

\section{Introduction}

When a liquid moves across a substrate surface, the line along which the free interface intersects with the surface is called the contact line. Free-surface flows with moving contact lines are commonly encountered in our daily lives. However, mathematical descriptions of such flows are beyond the capabilities of classical hydrodynamics. More precisely, if the classical no-slip condition is applied to a moving contact-line problem, a non-integrable stress singularity arises near the contact line (see, e.g., [5]).

The most common approach for avoiding this situation is to replace the no-slip condition with conditions that allow for slipping near the contact line (see, e.g., the review of slip models in Chap. 3 of [25]). This approach removes the above-mentioned stress singularity, but other issues remain unresolved. One such issue concerns the modeling of the dynamic contact angle. In slip models, the contact angle has to be prescribed, and in many cases it is assumed to be a function of the contact-line speed. However, a previous experimental study [2] suggests that the variation in the contact angle depends not only on the contact-line speed, but also on the flow field in the vicinity of the contact line. Another issue concerns the description of the flow kinematics. The experiments reported in [5] imply that a rolling motion arises in an actual flow. However, as reported in [24], two typical slip models (one that prescribes the fluid velocity on a solid surface and another that adopts the Navier slip condition) fail to reproduce the rolling motion.

(c) The Author(s) 2021. This article is licensed under a Creative Commons Attribution 4.0 International License, which permits use, sharing, adaptation, distribution and reproduction in any medium or format, as long as you give appropriate credit to the original author(s) and the source, provide a link to the Creative Commons licence, and indicate if changes were made. The images or other third party material in this article are included in the article's Creative Commons licence, unless indicated otherwise in a credit line to the material. If material is not included in the article's Creative Commons licence and your intended use is not permitted by statutory regulation or exceeds the permitted use, you will need to obtain permission directly from the copyright holder. To view a copy of this licence, visit http://creativecommons.org/licenses/by/4.0/. 
With the aim of overcoming the above-mentioned shortcomings of conventional models, Shikhmurzaev developed a new "interface formation model" [22]. Physically, the interface is considered to be a thin layer in which the density varies drastically because of the asymmetric action of intermolecular forces from the different phases. The interface formation model simulates this layer as a separate phase with zero thickness characterized by its surface density and surface velocity, where the surface density is related to the surface tension through an equation of state. This treatment of the interface makes it possible to describe the process of interface formation/disappearance occurring at the contact line and the variation in surface tension induced by these processes. The equations governing the relaxation process for the interface are derived by applying irreversible thermodynamics to the surface phase. The resultant equations consist of the balance laws for mass and momentum in the surface phase, which are coupled with the Navier-Stokes equations in the bulk phase by constitutive relations of the fluxes between the surface and bulk phases derived by applying Onsager's principle.

The main issues of dynamic wetting described above are consistently resolved by the interface formation model on the basis of the mechanism of interface formation and disappearance. This is the primary advantage of this model. The interface formation model naturally describes a flux at the contact line (see [22]), which is observed in the actual rolling motion. This flux is missing in the conventional models. The interface formation model relates the surface tension to the bulk velocity through boundary conditions. Thus, the contact angle is the response of the overall velocity field. In addition, the thermodynamics applied to the liquid-solid interface means that the no-slip condition is replaced with the generalized Navier slip condition. Thus, the interface formation model eliminates the non-integrable stress singularity at the contact line as a physical consequence.

As seen in [25], the interface formation model can describe various flows with moving contact lines, including dynamic wetting, cusp formation, and drop coalescence, in a unified way. This is another advantage of this model. In addition to Shikhmurzaev's studies (see [25] and the references therein), this model has been examined through applications to various flows using computational and asymptotic methods (see, e.g., $[1,3,4,7,8,16,17,26])$.

By contrast, we have relatively few mathematically rigorous results for the interface formation model. Mathematical studies on the solvability of free boundary problems for the Navier-Stokes (or Stokes) equations with moving contact lines have mainly been conducted for models with conventional boundary conditions (see, e.g., [9, 21, 28, 31, 32, 35]). If we restrict ourselves to the interface formation model, the solvability was studied in $[11,12]$; however, no situations involving contact lines were investigated in these studies, and to the best of the author's knowledge, there are no results on the solvability of Shikhmurzaev's full model describing flows with moving contact lines.

In this paper, we apply the interface formation model to the steadily advancing meniscus of an incompressible viscous fluid in a circular capillary tube, and prove that there exists an axially symmetric solution for low meniscus speeds. As stated in [24] or Sect. 2.5.2 in [25], for a hydrodynamic model describing the moving contact line to be physically valid, the solution of the model must remain finite near the contact line. We construct the above solution in weighted Hölder spaces to clarify the regularity of the solution near the contact line in the classical sense. As will be described in detail in the next section, we need to add one more condition on the contact line to ensure that the interface formation model is well 
posed. Following Shikhmurzaev's treatment in [24], a condition whereby the pressure is finite at the contact line is added. This treatment enables us to determine the contact angle as part of the solution without generating any singularity from the motion of the contact line. However, the case in which the contact angle is $\pi / 2$ is an exception (see the next section in this regard). The pressure diverges at the contact line when the contact angle is in the range $(\pi / 2, \pi)$, because the contact line is a ridge of the domain. It is shown that the singularity arising in this problem is caused only by the presence of the corner of the domain, and the regularity of the solution near the contact line is determined by the size of the contact angle.

The remainder of this paper is organized as follows. In Sect. 2, we formulate the problem. In Sect. 3, we introduce function spaces and state our main result. In Sect. 4, we give some results for the Stokes equations with the slip boundary condition defined in a sectorial domain. The regularity of the solution to our problem near the contact line is determined from the distribution of eigenvalues of the operator pencil associated with this model problem. In Sect. 5, we derive an estimate of the solution to the linearized problem. The estimate is obtained by combining Schauder's method with an exponential decay estimate for the Dirichlet integral obtained by means of differential inequalities techniques. In Sect. 6, we solve the nonlinear problem using the contraction mapping principle with the estimates established for the linearized problem. We construct a solution in a neighborhood of the rest state of the fluid. We assume that the static contact angle differs from 0 and $\pi$, and we do not consider the situation where the dynamic contact angle reaches 0 and $\pi$. In our problem, the location of the interface is not known a priori; hence, for technical convenience, we transform the problem onto a prescribed domain with fixed boundaries. However, as the contact angle is unknown in advance, the regularity of the solution near the contact line cannot be determined directly from the transformed problem. The regularity of the solution is determined after the contact angle has been found by solving the transformed problem. Finally, in Sect. 7, the overall conclusions from this study and future tasks are described.

\section{Formulation of the problem}

Consider the situation in which a meniscus is moving at a constant speed $W>0$ in an infinite circular tube $\Omega=\left\{\left|x^{\prime}\right| \equiv \sqrt{x_{1}^{2}+x_{2}^{2}}<1\right\}$. We assume that the total flux across an arbitrary cross-section of the part filled with the liquid is prescribed and given by $\rho|B| W$, where $\rho$ is the density of the liquid and $|B|$ is the area of the cross-section. In the formulation below, we assume that the flow is steady in the coordinate system moving with the meniscus.

First, we establish the governing equations. Let the region $\Omega_{h}=\left\{x_{3}<h\left(x^{\prime}\right), x^{\prime} \in B\right\}$ ( $B$ : $\left|x^{\prime}\right|<1$ ) be filled with a liquid and $\Gamma_{h}=\left\{x_{3}=h\left(x^{\prime}\right), x^{\prime} \in B\right\}$ be the free interface of the liquid. The interface is assumed to meet the surface $\partial \Omega$ at the contact line $M \equiv \bar{\Gamma}_{h} \cap \partial \Omega=\{x \in$ $\left.\partial \Omega \mid x_{3}=0\right\}$, and $\Sigma \equiv\left\{x \in \partial \Omega \mid x_{3}<0\right\}$ is the liquid-solid interface.

We assume that the flow over $\Omega_{h}$ is governed by the steady Navier-Stokes equations

$$
-v \Delta \mathbf{v}+(\mathbf{v} \cdot \nabla) \mathbf{v}+\nabla p=\mathbf{0}, \quad \nabla \cdot \mathbf{v}=0
$$

where $\mathbf{v}$ is the velocity, $p$ is the pressure, and $v$ is the kinematic viscosity. 
For $\Gamma_{h}$, where $\mathbf{n}$ is the unit outward normal to $\Gamma_{h}$, we consider the following equations:

$$
\begin{aligned}
& \Pi \mathbf{T}(\mathbf{v}, p) \mathbf{n}=\nabla_{\Gamma} \sigma_{1}, \quad \mathbf{n} \cdot \mathbf{T}(\mathbf{v}, p) \mathbf{n}=\sigma_{1} H, \\
& \nabla_{\Gamma} \cdot \rho_{1} \mathbf{v}_{1}=-\frac{\rho_{1}-\rho_{1, e}}{\tau_{1}}, \\
& \mathbf{v} \cdot \mathbf{n}=-\frac{\rho_{1}-\rho_{1, e}}{\rho \tau_{1}}, \quad \Pi\left(\mathbf{v}-\mathbf{v}_{1}\right)=-\chi \nabla_{\Gamma} \sigma_{1}, \\
& \sigma_{1}=\gamma\left(\rho_{0}-\rho_{1}\right),
\end{aligned}
$$

where $\mathbf{v}_{1}$ and $\rho_{1}$ are the velocity and surface density of the liquid-gas interface, respectively; $\mathbf{T}(\mathbf{v}, p)=2 \nu \mathbf{D}(\mathbf{v})-p \mathbf{I}$ is the stress tensor, where

$$
\mathbf{D}(\mathbf{v})=\frac{1}{2}\left(\frac{\partial v_{i}}{\partial x_{j}}+\frac{\partial v_{j}}{\partial x_{i}}\right)_{i, j=1,2,3}
$$

is the strain-rate tensor; $H$ is twice the mean curvature of $\Gamma_{h}$, which is assumed to be negative where $\Omega_{h}$ is convex; $\Pi$ is the projection operator onto the tangent plane of $\Gamma_{h}$; $\nabla_{\Gamma}$ is the gradient operator defined on $\Gamma_{h}$; and $\rho_{1, e}, \tau_{1}, \chi, \gamma$, and $\rho_{0}$ are positive constants. In particular, $\rho_{1, e}$ is the equilibrium surface density.

For $\Sigma$, we assume that

$$
\begin{aligned}
& \Pi \mathbf{T}(\mathbf{v}, p) \mathbf{n}-\frac{1}{2} \nabla_{\Sigma} \sigma_{2}=-\beta \Pi(\mathbf{v}-\mathbf{W}), \\
& \nabla_{\Sigma} \cdot \rho_{2} \mathbf{v}_{2}=-\frac{\rho_{2}-\rho_{2, e}}{\tau_{2}}, \\
& \mathbf{v} \cdot \mathbf{n}=-\frac{\rho_{2}-\rho_{2, e}}{\rho \tau_{2}}, \quad \Pi \mathbf{v}_{2}=\frac{1}{2} \Pi(\mathbf{v}+\mathbf{W})+\alpha \nabla_{\Sigma} \sigma_{2}, \\
& \sigma_{2}=\gamma\left(\rho_{0}-\rho_{2}\right),
\end{aligned}
$$

where $\mathbf{v}_{2}$ and $\rho_{2}$ are the velocity and surface density of the liquid-solid interface, respectively; $\mathbf{W}=(0,0,-W) ; \mathbf{n}, \Pi$, and $\nabla_{\Sigma}$ are defined for $\Sigma$ in the same way as for $\Gamma_{h}$; and $\rho_{2, e}$, $\tau_{2}, \alpha$, and $\beta$ are positive constants. In particular, $\rho_{2, e}$ is the equilibrium surface density.

In regard to the interfaces, (2) and (6) represent the momentum balance, (3) and (7) represent the mass balance, (5) and (9) are equations of state, and (4) and (8) are phenomenological laws between fluxes and thermodynamic forces arising in the entropy production rate of the interface (for details of the derivation, see [22, 23, 25]).

Remark 1 In the present paper, we assume that the right-hand sides of $(4)^{1}$ and $(8)^{1}$ vanish. In the original version of the interface formation model, Shikhmurzaev derived these conditions by assuming that the effect of mass exchange on the bulk is negligible (see, e.g., [23]). However, as revealed in [24], these terms play an essential role in describing the flow in the immediate vicinity of the contact lines more accurately. We have not yet succeeded in proving the solvability of the same problem when the right-hand sides of $(4)^{1}$ and $(8)^{1}$ do not vanish. The main difficulty lies in the derivation of an inequality corresponding to (54), which is essential in proving the solvability of the linear problem. 
On $M$, we assume the conservation of mass, i.e.,

$$
\rho_{1} \mathbf{v}_{1} \cdot \mathbf{e}_{1}+\rho_{2} \mathbf{v}_{2} \cdot \mathbf{e}_{2}=0
$$

where $\mathbf{e}_{1}$ and $\mathbf{e}_{2}$ are unit normal vectors to $M$ that are tangential to $\Gamma_{h}$ and $\Sigma$, respectively. We also assume the following force balance:

$$
\sigma_{1} \cos \theta_{d}=\sigma_{s g}-\sigma_{2}
$$

where $\theta_{d}$ is the contact angle determined by $\cos \theta_{d}=\mathbf{e}_{1} \cdot \mathbf{e}_{2}$, and $\sigma_{s g}$ is the solid-gas surface tension, assumed to be a nonnegative constant.

Now, let us formulate our problem. After eliminating the surface velocity $\mathbf{v}_{1}$ with the aid of $(4)^{2}$ and $\mathbf{v}_{2}$ with the aid of $(8)^{2}$, the above equations can be written as follows:

$$
\begin{aligned}
& -v \Delta \mathbf{v}+(\mathbf{v} \cdot \nabla) \mathbf{v}+\nabla p=\mathbf{0}, \quad \nabla \cdot \mathbf{v}=0 \quad \text { in } \Omega_{h}, \\
& \left\{\begin{array}{l}
\Pi \mathbf{T}(\mathbf{v}, p) \mathbf{n}+\gamma \nabla_{\Gamma} \rho_{1}=\mathbf{0}, \quad \mathbf{n} \cdot \mathbf{T}(\mathbf{v}, p) \mathbf{n}=\sigma_{1} H, \\
-\chi \gamma \nabla_{\Gamma} \cdot \rho_{1} \nabla_{\Gamma} \rho_{1}+\nabla_{\Gamma} \cdot \rho_{1} \mathbf{v}=-\frac{\rho_{1}-\rho_{1, e}}{\tau_{1}}, \\
\mathbf{v} \cdot \mathbf{n}=0 \quad \text { on } \Gamma_{h},
\end{array}\right. \\
& \left\{\begin{array}{l}
\Pi \mathbf{T}(\mathbf{v}, p) \mathbf{n}+\frac{\gamma}{2} \nabla_{\Sigma} \rho_{2}=-\beta(\mathbf{v}-\mathbf{W}), \\
-\alpha \gamma \nabla_{\Sigma} \cdot \rho_{2} \nabla_{\Sigma} \rho_{2}+\frac{1}{2} \nabla_{\Sigma} \cdot \rho_{2}(\mathbf{v}+\mathbf{W})=-\frac{\rho_{2}-\rho_{2, e}}{\tau_{2}}, \\
\mathbf{v} \cdot \mathbf{n}=0 \quad \text { on } \Sigma,
\end{array}\right. \\
& \rho_{1}\left(\mathbf{v}-\chi \gamma \nabla_{\Gamma} \rho_{1}\right) \cdot \mathbf{e}_{1} \\
& =-\rho_{2}\left\{\frac{1}{2}(\mathbf{v}+\mathbf{W})-\alpha \gamma \nabla_{\Sigma} \rho_{2}\right\} \cdot \mathbf{e}_{2} \quad \text { on } M, \\
& \left(\rho_{0}-\rho_{1}\right) \cos \theta_{d}=\sigma_{3}-\left(\rho_{0}-\rho_{2}\right)\left(\sigma_{3}=\sigma_{s g} / \gamma\right) \quad \text { on } M,
\end{aligned}
$$

and

$$
\int_{B} v_{3}\left(x^{\prime}, x_{3}\right) d x^{\prime}=0 \quad\left(x_{3} \leq 0\right) .
$$

Further, we assume that the liquid-solid interface reaches its equilibrium state and that the flow is fully developed in the far field, and we impose the following condition:

$$
\left(\mathbf{v}, \rho_{2}\right) \longrightarrow\left(\mathbf{V}, \rho_{2, e}\right) \quad\left(x_{3} \longrightarrow-\infty\right)
$$

Here, $\mathbf{V}$ is the Poiseuille flow given by the pair $(\mathbf{V}, P)$, where

$$
\left\{\begin{array}{l}
\mathbf{V}=\left(0,0,-W-W \frac{2}{1+4 v / \beta}\left(\left|x^{\prime}\right|^{2}-1-\frac{2 v}{\beta}\right)\right) \\
P=-W \frac{8 v}{1+4 v / \beta} x_{3} .
\end{array}\right.
$$

This Poiseuille flow was derived in [34].

As seen in $(13)^{3}$ and $(14)^{2}$, the surface densities $\rho_{1}$ and $\rho_{2}$ satisfy second-order elliptic partial differential equations. Therefore, from a mathematical point of view, we need 
two boundary conditions on $M$ to determine the surface densities using these equations. However, between conditions (15) and (16) on $M$, we can use only (15) for that purpose, because condition (16) is used to determine the contact angle. To use (16) as a boundary condition to determine the surface densities, we need to prescribe the contact angle, but doing so would eliminate the main advantage of this model. This "lack of a condition" was first pointed out in [1].

When the contact line is moving, the term $\beta \mathbf{W}$ arises in the generalized Navier condition (6). Shikhmurzaev [24] adds condition (50) (see [24]) to remove a pressure singularity caused by the above term and constructs an asymptotic solution without a pressure singularity. We follow the above treatment and assume his condition (50) in the following equivalent form:

$$
\beta \mathbf{W} \cdot \mathbf{e}_{2}-\frac{\gamma}{2} \nabla_{\Sigma} \rho_{2} \cdot \mathbf{e}_{2}-\gamma \nabla_{\Gamma} \rho_{1} \cdot \mathbf{e}_{1}=0 \quad \text { on } M
$$

We can also find this condition from the right-hand side of conditions $(13)^{1,4}$ and $(14)^{1,3}$ by setting $a_{1}=\gamma \nabla_{\Gamma} \rho_{1} \cdot \mathbf{e}_{1}, b_{1}=-\left(\frac{\gamma}{2} \nabla \rho_{2}+\beta \Pi(\mathbf{v}-\mathbf{W})\right) \cdot \mathbf{e}_{2}, a_{2}=b_{2}=0$ and applying condition (26) to these terms (in this case the problem domain is not a sector, so the term $\frac{\partial a_{2}}{\partial \tau}$ is replaced by $\left.\frac{\partial a_{2}}{\partial \tau}-\mathbf{v} \cdot \frac{\partial \mathbf{n}}{\partial \tau}\right)$.

Remark 2 Another approach for adding a condition at the contact line can be found in [1], where a boundary condition is added by accounting for the entropy produced at the contact line. Shikhmurzaev disagrees with this modeling approach from a physical point of view (see Sect. 4.3.5.3 in [25]).

Condition (20) ensures that the compatibility condition corresponding to (34) is satisfied for problem (91), which makes it possible to construct a solution without a singularity at the contact line when $\theta_{d}<\pi / 2$. However, when $\theta_{d}>\pi / 2$, the pressure becomes singular because the model problem (23) has singular eigensolutions. When $\theta_{d}=\pi / 2$, in addition to (20), the condition that

$$
\beta \mathbf{W} \cdot \mathbf{e}_{2}-\frac{\gamma}{2} \nabla_{\Sigma} \rho_{2} \cdot \mathbf{e}_{2}+\gamma \nabla_{\Gamma} \rho_{1} \cdot \mathbf{e}_{1}=0 \quad \text { on } M,
$$

which comes from condition (30), has to be imposed for the pressure to be regular at the contact line. However, if we further assume condition (21), our problem becomes overdetermined. In addition, it is impossible to impose a condition for a specific contact angle in advance for a problem where the contact angle is not known. For these reasons, we cannot construct a regular solution when $\theta_{d}=\pi / 2$.

We hereafter denote the problem consisting of equations (12)-(20) for unknown functions $\left(\mathbf{v}, p, \rho_{1}, \rho_{2}, h\right)$ as problem $(\mathcal{P})$.

\section{Main result}

To state our main result, we first introduce the function spaces used throughout this paper. Let $D$ be a domain in $\mathbb{R}^{n}, l$ be a nonnegative integer, and $\alpha \in(0,1)$. We use $C^{l+\alpha}(D)$ to denote the space of functions $f(x), x \in D$ with the norms

$$
|f|_{D}^{(l+\alpha)} \equiv \sum_{|m| \leq l}\left|\partial_{x}^{m} f\right|_{D}^{(0)}+[f]_{D}^{(l+\alpha)}, \quad|f|_{D}^{(0)} \equiv \sup _{x \in D}|f(x)|,
$$




$$
[f]_{D}^{(l+\alpha)} \equiv \sum_{|m|=l}\left[\partial_{x}^{m} f\right]_{D}^{(\alpha)} \equiv \sup _{\substack{x, y \in D \\ x \neq y}} \sum_{|m|=l} \frac{\left|\partial_{x}^{m} f(x)-\partial_{y}^{m} f(y)\right|}{|x-y|^{\alpha}}
$$

where

$$
|m|=\sum_{i=1}^{n} m_{i} \quad\left(m_{i}(\geq 0) \in \mathbb{Z}\right), \quad \partial_{x}^{m}=\frac{\partial^{|m|}}{\partial_{x_{1}}^{m_{1}} \cdots \partial_{x_{n}}^{m_{n}}} .
$$

Below, we introduce weighted Hölder spaces. Let $s \in \mathbb{R}$ and $M \subset \partial D$ be a closed set. Using $\stackrel{\circ}{C}_{s}^{l+\alpha}(D, M)$, we denote the function space with the norm

$$
\langle f\rangle_{s, D, M}^{(l+\alpha)} \equiv \sum_{0 \leq|m| \leq l}\left|\rho^{|m|-s} \partial_{x}^{m} f\right|_{D}^{(0)}+[f]_{s, D, M}^{(l+\alpha)}
$$

where

$$
[f]_{s, D, M}^{(l+\alpha)} \equiv \sum_{|m|=l} \sup _{x \in D} \rho^{l+\alpha-s}(x) \sup _{y \in K(x)} \frac{\left|\partial_{x}^{m} f(x)-\partial_{y}^{m} f(y)\right|}{|x-y|^{\alpha}},
$$

$K(x)=\left\{y \in D|| y-x \mid<\frac{1}{2} \rho(x)\right\}$, and $\rho(x)=\operatorname{dist}(x, M)$.

Let $s(\notin \mathbb{Z}) \in(0, l+\alpha]$. Let $C_{s}^{l+\alpha}(D, M)$ be the function space with the norm

$$
|f|_{s, D, M}^{(l+\alpha)} \equiv|f|_{D}^{(s)}+\sum_{s<|m| \leq l}\left|\rho^{|m|-s} \partial_{x}^{m} f\right|_{D}^{(0)}+[f]_{s, D, M}^{(l+\alpha)}
$$

For $s<0$, we assume that $C_{s}^{l+\alpha}(D, M)=\stackrel{\circ}{C}_{s}^{l+\alpha}(D, M)$.

Further, we introduce a weighted Hölder space defined on the infinite semi-cylinder $\Omega_{h}$. Let $\mu>0, \Omega_{t}=\left\{x \in \Omega_{h} \mid x_{3}<-t\right\}$, and $\Omega_{0, t}=\left\{x \in \Omega_{h} \mid x_{3}>-t\right\}$, where $t>0$. Let $C_{s}^{l+\alpha, \mu}\left(\Omega_{h}, M\right)$ be the function space with the norm

$$
|f|_{s, \Omega_{h}, M}^{(l+\alpha, \mu)} \equiv|f|_{s, \Omega_{0,2}, M}^{(l+\alpha)}+|f|_{\Omega_{1}}^{(l+\alpha, \mu)}, \quad|f|_{\Omega_{1}}^{(l+\alpha, \mu)} \equiv\left|e^{-\mu x_{3}} f\right|_{\Omega_{1}}^{(l+\alpha)} .
$$

We also introduce the space $C_{s}^{l+\alpha, \mu}(\Sigma, M)$ of functions defined on the lateral surface $\Sigma$, which is defined in the same manner as $C_{s}^{l+\alpha, \mu}\left(\Omega_{h}, M\right)$, except that $\Omega_{h}$ is replaced by $\Sigma$.

Finally, let $W_{2}^{l}(D)$ be the function space with the norm

$$
\|f\|_{2, D}^{(l)} \equiv \sum_{|m| \leq l}\left\|\partial_{x}^{m} f\right\|_{2, D}, \quad\|f\|_{2, D} \equiv\left(\int_{D}|f(x)|^{2} d x\right)^{\frac{1}{2}} .
$$

The following is our main result.

Theorem 1 Let $\lambda_{0}>0$ be the smallest noninteger solution of the equation

$$
\sin (1+\lambda) \theta_{d} \cdot \sin (1-\lambda) \theta_{d}=0 .
$$

When $\theta_{d}=\pi / 2$, we assume that $\lambda_{0}=1$. Let $0<\alpha<1$ and $s$ satisfy the conditions $s \notin \mathbb{Z}$, $0<s \leq 2+\alpha$, and $0<s<\min \left(\pi / \theta_{d}, \lambda_{0}\right)$. Assume that $2 \rho_{2, e} \alpha-\rho_{1, e} \chi \neq 0$ and $\left|\left(\sigma_{s g}-\bar{\sigma}_{2}\right) / \bar{\sigma}_{1}\right|<$ 
$\delta_{0}<1$ for a constant $\delta_{0}$, where $\bar{\sigma}_{1}=\gamma\left(\rho_{0}-\rho_{1, e}\right), \bar{\sigma}_{2}=\gamma\left(\rho_{0}-\rho_{2, e}\right)$. Then, if $W>0$ is sufficiently small, for some constant $\mu>0$, problem $(\mathcal{P})$ has an axisymmetric solution $\left(\mathbf{v}, p, \rho_{1}, \rho_{2}, h\right)$ with the following properties:

- The angular component of $\mathbf{v}$ around the $x_{3}$-axis vanishes;

- $\theta_{d} \neq 0, \pi$

- The solution belongs to the following classes:

$$
\begin{aligned}
& \mathbf{v}-\zeta \mathbf{V} \in C_{s}^{2+\alpha, \mu}\left(\Omega_{h}, M\right), \quad \nabla(p-\zeta P) \in C_{s-2}^{\alpha, \mu}\left(\Omega_{h}, M\right), \quad p \in C_{s-1}^{1+\alpha}\left(\Omega_{0,1}, M\right), \\
& \rho_{1} \in C_{s+1}^{3+\alpha}\left(\Gamma_{h}, M\right), \quad \rho_{2}-\rho_{2, e} \in C_{s+1}^{3+\alpha, \mu}(\Sigma, M), \quad h \in C_{s+1}^{3+\alpha}(B, M),
\end{aligned}
$$

where $\zeta$ is the function given in (75).

Remark 3 The condition $2 \rho_{2, e} \alpha-\rho_{1, e} \chi \neq 0$ is required for conditions (15) and (20) to be written as two independent conditions $(91)^{9,10}$.

\section{Auxiliary problem in a sectorial domain}

Assume that $0<\theta<\pi$. Let $d_{\theta}, \gamma_{\theta}, \gamma_{0}$, and $M$ be defined by $\left\{x_{2}>0, x_{1}>\frac{x_{2}}{\tan \theta}\right\},\left\{x_{2}>0, x_{2}=\right.$ $\left.x_{1} \tan \theta\right\},\left\{x_{1}>0, x_{2}=0\right\}$, and $\bar{\gamma}_{\theta} \cap \bar{\gamma}_{0}$, respectively. When $\theta=\pi / 2, d_{\theta}$ and $\gamma_{\theta}$ are replaced by $\left\{x_{2}>0, x_{1}>0\right\}$ and $\left\{x_{2}>0, x_{1}=0\right\}$, respectively. We consider the following problem:

$$
\begin{cases}-v \Delta \mathbf{u}+\nabla q=\mathbf{f}, & \nabla \cdot \mathbf{u}=g \quad \text { in } d_{\theta}, \\ 2 \nu \mathbf{D}(\mathbf{u}) \mathbf{n} \cdot \tau=a_{1}, & \mathbf{u} \cdot \mathbf{n}=a_{2} \quad \text { on } \gamma_{0}, \\ 2 \nu \mathbf{D}(\mathbf{u}) \mathbf{n} \cdot \tau=b_{1}, & \mathbf{u} \cdot \mathbf{n}=b_{2} \quad \text { on } \gamma_{\theta},\end{cases}
$$

where $\tau=(1,0)$ on $\gamma_{0},(\cos \theta, \sin \theta)$ on $\gamma_{\theta}$, and $\mathbf{n}=\left(-\tau_{2}, \tau_{1}\right)$.

The following lemma for the above problem will be used in the next section. As we can prove this lemma in a standard manner (see, e.g., [29]), the proof is omitted.

Lemma 1 Let $\lambda_{0}>0$ be the smallest noninteger solution of the equation

$$
\sin (1+\lambda) \theta \cdot \sin (1-\lambda) \theta=0
$$

If $\theta=\pi / 2$, assume that $\lambda_{0}=1$. Assume that $0<\alpha<1, s \notin \mathbb{Z}, 0<s<\lambda_{0}$, and $s \leq 2+\alpha$. Further, assume that $\mathbf{f} \in C_{s-2}^{\alpha}\left(d_{\theta}, M\right), g \in C_{s-1}^{1+\alpha}\left(d_{\theta}, M\right), a_{1} \in C_{s-1}^{1+\alpha}\left(\gamma_{0}, M\right), a_{2} \in C_{s}^{2+\alpha}\left(\gamma_{0}, M\right)$, $b_{1} \in C_{s-1}^{1+\alpha}\left(\gamma_{\theta}, M\right)$, and $b_{2} \in C_{s}^{2+\alpha}\left(\gamma_{\theta}, M\right)$, and that their supports are compact. Additionally, assume that the condition

$$
\int_{d_{\theta}} g d x=-\int_{\gamma_{0}} a_{2} d l+\int_{\gamma_{\theta}} b_{2} d l
$$

is satisfied, and, if $\lambda_{0}>1$, further assume that the following compatibility condition is satisfied:

$$
a_{1}-\left.2 v \frac{\partial a_{2}}{\partial \tau}\right|_{M}=b_{1}-\left.2 v \frac{\partial b_{2}}{\partial \tau}\right|_{M} .
$$


Then (23) has a unique solution $(\mathbf{u}, \nabla q) \in C_{s}^{2+\alpha}\left(d_{\theta}, M\right) \times C_{s-2}^{\alpha}\left(d_{\theta}, M\right)$ that satisfies the estimate

$$
\begin{aligned}
|\mathbf{u}|_{s, d_{\theta}, M}^{(2+\alpha)}+|\nabla q|_{s-2, d_{\theta}, M}^{(\alpha)} \leq & C\left(|\mathbf{f}|_{s-2, d_{\theta}, M}^{(\alpha)}+|g|_{s-1, d_{\theta}, M}^{(1+\alpha)}\right. \\
& \left.+\left|a_{1}\right|_{s-1, \gamma_{0}, M}^{(1+\alpha)}+\left|a_{2}\right|_{s, \gamma_{0}, M}^{(2+\alpha)}+\left|b_{1}\right|_{s-1, \gamma_{\theta}, M}^{(1+\alpha)}+\left|b_{2}\right|_{s, \gamma_{\theta}, M}^{(2+\alpha)}\right)
\end{aligned}
$$

for a constant $C>0$ that is independent of the data.

Rewrite problem (23) with $v=1$ in the polar coordinates $(r, \phi)$ defined by $x_{1}=$ $r \cos \phi, x_{2}=r \sin \phi$, then apply the Mellin transform with respect to $r$ :

$$
\mathcal{M}[f](\lambda)=\tilde{f}(\lambda) \equiv \int_{0}^{\infty} r^{-\lambda-1} f(r) d r
$$

Thus, we have the following system of ordinary differential equations for $u_{r}=u_{1} \cos \phi+$ $u_{2} \sin \phi, u_{\phi}=-u_{1} \sin \phi+u_{2} \cos \phi$ :

$$
\left\{\begin{array}{l}
-\tilde{u}_{r}^{\prime \prime}+\left(1-\lambda^{2}\right) \tilde{u}_{r}+2 \tilde{u}_{\phi}^{\prime}+(\lambda-1) \tilde{q}=\tilde{f}_{1}, \\
-\tilde{u}_{\phi}^{\prime \prime}+\left(1-\lambda^{2}\right) \tilde{u}_{\phi}-2 \tilde{u}_{r}^{\prime}+\tilde{q}^{\prime}=\tilde{f}_{2}, \\
\tilde{u}_{\phi}^{\prime}+(\lambda+1) \tilde{u}_{r}=\tilde{g}, \\
(\lambda-1) \tilde{u}_{\phi}+\left.\tilde{u}_{r}^{\prime}\right|_{\phi=0}=\tilde{a}_{1},\left.\quad \tilde{u}_{\phi}\right|_{\phi=0}=\tilde{a}_{2}, \\
(\lambda-1) \tilde{u}_{\phi}+\left.\tilde{u}_{r}^{\prime}\right|_{\phi=\theta}=\tilde{b}_{1},\left.\quad \tilde{u}_{\phi}\right|_{\phi=\theta}=\tilde{b}_{2} .
\end{array}\right.
$$

We denote the operator of this boundary value problem by $\mathcal{U}(\lambda)$. If $\lambda$ is not a root of (24), this problem has a unique solution. The roots of (24) are called eigenvalues of problem (28). A nontrivial solution corresponding to each eigenvalue is called an eigenvector.

The asymptotic formula (29) in the following lemma describes the singularity of solutions to problem (23). (For the derivation, see, e.g., [10, 15].)

Lemma 2 Let $\stackrel{\circ}{S}_{s}^{\alpha} \equiv \stackrel{\circ}{C}_{s}^{2+\alpha}\left(d_{\theta}\right) \times \stackrel{\circ}{C}_{s-1}^{1+\alpha}\left(d_{\theta}\right)$ and $\stackrel{\circ}{D}_{s}^{\alpha} \equiv \stackrel{\circ}{C}_{s-2}^{\alpha}\left(d_{\theta}\right) \times \stackrel{\circ}{C}_{s-1}^{1+\alpha}\left(d_{\theta}\right) \times \stackrel{\circ}{C}_{s-1}^{1+\alpha}\left(\gamma_{0}\right) \times$ $\stackrel{\circ}{C}_{s}^{2+\alpha}\left(\gamma_{0}\right) \times \stackrel{\circ}{C}_{s-1}^{1+\alpha}\left(\gamma_{\theta}\right) \times \stackrel{\circ}{C}_{s}^{2+\alpha}\left(\gamma_{\theta}\right)$. Assume that $s_{1}, s_{2}\left(0<s_{1}<s_{2}\right) \notin \mathbb{Z}$ are not roots of (24). Let $\left(\mathbf{f}, g, a_{1}, a_{2}, b_{1}, b_{2}\right) \in \stackrel{\circ}{D}_{s_{1}}^{\alpha} \cap \stackrel{\circ}{D}_{s_{2}}^{\alpha}$, and let $\lambda_{1}, \ldots, \lambda_{n}$ be the eigenvalues of problem (28) that exist in the range $\left(s_{1}, s_{2}\right)$. Then the solutions $\left(\mathbf{u}_{1}, q_{1}\right) \in \stackrel{\circ}{S}_{s_{1}}^{\alpha}$ and $\left(\mathbf{u}_{2}, q_{2}\right) \in \stackrel{\circ}{S}_{s_{2}}^{\alpha}$ of problem (23) are related by the formula

$$
\left(\mathbf{u}_{1}, q_{1}\right)=\sum_{j=1}^{n} c_{j}\left(r^{\lambda_{j}} \mathbf{u}\left(\lambda_{j}\right), r^{\lambda_{j}-1} q\left(\lambda_{j}\right)\right)+\left(\mathbf{u}_{2}, q_{2}\right)
$$

where $c_{j}$ are constants depending on the data, and $\left(\mathbf{u}\left(\lambda_{j}\right), q\left(\lambda_{j}\right)\right)$ is the eigenvector of problem (28) corresponding to eigenvalue $\lambda_{j}$.

From (29), we can see how $q$ behaves near the corner $M$. Let $\lambda_{1}$ in (29) be the smallest positive eigenvalue. When $0<\theta<\pi / 2, \lambda_{1}$ is greater than 1 , which indicates that $q$ remains bounded near $M$. In contrast, when $\pi / 2<\theta<\pi, q$ diverges at $M$.

When $\theta=\pi / 2$, all nonnegative eigenvalues are given by $\lambda=2 k+1(k=0,1,2, \ldots)$. These eigenvalues are all double roots, and the corresponding eigenvectors are given 
by $(\mathbf{u}(\lambda), q(\lambda))=(\cos 2(k+1) \phi,-\sin 2(k+1) \phi, 0)$ and $(-k \cos 2 k \phi,(k+1) \sin 2 k \phi,-2(2 k+$ $1) \cos 2 k \phi)$. If the associated eigenvector $(\mathbf{v}(\lambda), p(\lambda))$ defined as a solution of the equation

$$
\mathcal{U}(\lambda)(\mathbf{v}(\lambda), p(\lambda))+\mathcal{U}^{\prime}(\lambda)(\mathbf{u}(\lambda), q(\lambda))=(\mathbf{0}, 0)
$$

exists for $\lambda_{j}$, the term $c_{j}\left(r^{\lambda_{j}} \mathbf{u}\left(\lambda_{j}\right), r^{\lambda_{j}-1} q\left(\lambda_{j}\right)\right)$ in (29) is replaced by

$$
c_{j}\left\{\left(r^{\lambda_{j}} \mathbf{v}\left(\lambda_{j}\right), r^{\lambda_{j}-1} p\left(\lambda_{j}\right)\right)+\log r\left(r^{\lambda_{j}} \mathbf{u}\left(\lambda_{j}\right), r^{\lambda_{j}-1} q\left(\lambda_{j}\right)\right)\right\} .
$$

However, elementary calculations show that no associated eigenvectors exist for all the eigenvalues given above. This implies that the solution to problem (23) is regular near the corner $M$. Indeed, we can obtain the following result in a similar manner to Sect. 6 of [20].

Lemma 3 Assume that $0<\alpha<1$. Assume that $\mathbf{f} \in C^{\alpha}\left(d_{\theta}\right), g \in C^{1+\alpha}\left(d_{\theta}\right), a_{1} \in C^{1+\alpha}\left(\gamma_{0}\right), a_{2} \in$ $C^{2+\alpha}\left(\gamma_{0}\right), b_{1} \in C^{1+\alpha}\left(\gamma_{\theta}\right)$, and $b_{2} \in C^{2+\alpha}\left(\gamma_{\theta}\right)$, and that their supports are compact. Further, assume that compatibility conditions (25), (26), and

$$
\left.a_{1}\right|_{M}=-\left.b_{1}\right|_{M}
$$

hold.

Then (23) has a unique solution $(\mathbf{u}, \nabla q) \in C^{2+\alpha}\left(d_{\theta}\right) \times C^{\alpha}\left(d_{\theta}\right)$ that satisfies the estimate

$$
\begin{aligned}
|\mathbf{u}|_{d_{\theta}}^{(2+\alpha)}+|\nabla q|_{d_{\theta}}^{(\alpha)} \leq & C\left(|\mathbf{f}|_{d_{\theta}}^{(\alpha)}+|g|_{d_{\theta}}^{(1+\alpha)}\right. \\
& \left.+\left|a_{1}\right|_{\gamma_{0}}^{(1+\alpha)}+\left|a_{2}\right|_{\gamma_{0}}^{(2+\alpha)}+\left|b_{1}\right|_{\gamma_{\theta}}^{(1+\alpha)}+\left|b_{2}\right|_{\gamma_{\theta}}^{(2+\alpha)}\right)
\end{aligned}
$$

for a constant $C>0$ that is independent of the data.

\section{Linear problem}

In this section, we consider the following problem:

$$
\left\{\begin{array}{l}
-v \Delta \mathbf{v}+\nabla p=\mathbf{f}, \quad \nabla \cdot \mathbf{v}=g \quad \text { in } \Omega, \\
2 v \Pi \mathbf{D}(\mathbf{v}) \mathbf{n}+\gamma \nabla_{\Gamma} \rho_{1}=\mathbf{a}, \quad \mathbf{v} \cdot \mathbf{n}=a_{3}, \\
-\kappa_{1} \nabla_{\Gamma}^{2} \rho_{1}+\chi_{1} \rho_{1}+\nabla_{\Gamma} \cdot \mathbf{v}=h_{1} \quad \text { on } \Gamma, \\
2 v \Pi \mathbf{D}(\mathbf{v}) \mathbf{n}+\beta \Pi \mathbf{v}+\frac{\gamma}{2} \nabla_{\Sigma} \rho_{2}=\mathbf{b}, \quad \mathbf{v} \cdot \mathbf{n}=b_{3}, \\
-\kappa_{2} \nabla_{\Sigma}^{2} \rho_{2}+\chi_{2} \rho_{2}+\frac{1}{2} \nabla_{\Sigma} \cdot \mathbf{v}=h_{2} \quad \text { on } \Sigma, \\
\left(\mathbf{v}-\kappa_{1} \nabla_{\Gamma} \rho_{1}\right) \cdot \mathbf{e}_{1}=m_{1}, \\
\left(\frac{1}{2} \mathbf{v}-\kappa_{2} \nabla_{\Sigma} \rho_{2}\right) \cdot \mathbf{e}_{2}=m_{2} \quad \text { on } M, \\
\mathbf{v} \longrightarrow \mathbf{0}, \quad \rho_{2} \longrightarrow 0 \quad \text { as } x_{3} \longrightarrow-\infty,
\end{array}\right.
$$

where $\nu, \gamma, \kappa_{1}, \kappa_{2}, \beta, \chi_{1}$, and $\chi_{2}$ are positive constants, and $\Omega$ is defined for a given function $h \in C_{s+1}^{3+\alpha}(B)$ in the same manner as $\Omega_{h}$ was defined in Sect. 2. Similarly, $\Gamma, \Sigma, M, \mathbf{n}, \mathbf{e}_{1}, \mathbf{e}_{2}$, $\nabla_{\Gamma}$, and $\nabla_{\Sigma}$ are defined in the same way as in Sect. 2. We assume that the domain $\Omega$ and the data $\mathbf{f}, g, \mathbf{a}, a_{3}, \mathbf{b}, b_{3}, h_{1}, h_{2}, m_{1}$, and $m_{2}$ do not depend on the rotation angle $\phi$ around the $x_{3}$-axis, and the $\phi$-components of $\mathbf{f}, \mathbf{a}$, and $\mathbf{b}$ vanish in the cylindrical coordinates $(r, \phi, z)$ 
defined by $\left(x_{1}, x_{2}, x_{3}\right)=(r \cos \phi, r \sin \phi, z)$. We denote the contact angle between $\Gamma$ and $\Sigma$ as $\theta$ and assume that $\theta \neq 0, \pi$.

The following is the main result in this section.

Theorem 2 Let $0<\alpha<1$ and $s$ be a constant satisfying the conditions $s \leq 2+\alpha, s \notin$ $\mathbb{Z}$, and $0<s<\min \left(\lambda_{0}, \pi / \theta\right)$, where $\lambda_{0}$ is the constant given in Lemma 1 . Assume that $\mathbf{f} \in C_{s-2}^{\alpha, \mu}(\Omega, M), g \in C_{s-1}^{1+\alpha, \mu}(\Omega, M), \mathbf{a} \in C_{s-1}^{1+\alpha}(\Gamma, M), a_{3} \in C_{s}^{2+\alpha}(\Gamma, M), \mathbf{b} \in C_{s-1}^{1+\alpha, \mu}(\Sigma, M)$, $b_{3} \in C_{s}^{2+\alpha, \mu}(\Sigma, M), h_{1} \in C_{s-1}^{1+\alpha}(\Gamma, M), h_{2} \in C_{s-1}^{1+\alpha, \mu}(\Sigma, M)$, and $m_{1}, m_{2} \in C^{s}(M)$. In addition, the following condition holds:

$$
\int_{\Omega} g d x=\int_{\Gamma} a_{3} d S+\int_{\Sigma} b_{3} d S
$$

For the case $\theta<\frac{\pi}{2}$, we also assume the compatibility condition at the contact line $M$ :

$$
\begin{aligned}
0= & \mathbf{b} \cdot \mathbf{e}_{2}-\left.\beta \mathbf{v}\right|_{M} \cdot \mathbf{e}_{2}+\frac{\gamma}{2 \kappa_{2}}\left(m_{2}-\left.\frac{1}{2} \mathbf{v}\right|_{M} \cdot \mathbf{e}_{2}\right)+\mathbf{a} \cdot \mathbf{e}_{1} \\
& +\frac{\gamma}{\kappa_{1}}\left(m_{1}-\left.\mathbf{v}\right|_{M} \cdot \mathbf{e}_{1}\right)+2 v \partial_{z} b_{3}+2 v\left(\frac{\partial a_{3}}{\partial \tau}-\left.\mathbf{v}\right|_{M} \cdot \frac{\partial \mathbf{n}}{\partial \tau}\right)
\end{aligned}
$$

with

$$
\left.\mathbf{v}\right|_{M}=\left.\left(v_{r}, v_{\phi}, v_{z}\right)\right|_{M}=\left(b_{3}, v_{\phi}, h^{\prime}(1) b_{3}+\sqrt{1+h^{\prime}(1)^{2}} a_{3}\right),
$$

where $\tau$ is given by $\left(\frac{1}{\sqrt{1+h^{\prime}(r)^{2}}}, 0, \frac{h^{\prime}(r)}{\sqrt{1+h^{\prime}(r)^{2}}}\right)$ in the cylindrical coordinates introduced above. Then there exists a constant $\mu>0$ such that problem (32) has a unique axisymmetric solution with the following properties: the $\phi$-component of $\mathbf{v}$ vanishes, $\mathbf{v} \in C_{s}^{2+\alpha, \mu}(\Omega, M)$, $\nabla p \in C_{s-2}^{\alpha, \mu}(\Omega, M), \rho_{1} \in C_{s+1}^{3+\alpha}(\Gamma, M)$, and $\rho_{2} \in C_{s+1}^{3+\alpha, \mu}(\Sigma, M)$, and these functions satisfy the estimate

$$
\begin{aligned}
&|\mathbf{v}|_{s, \Omega, M}^{(2+\alpha, \mu)}+|\nabla p|_{s-2, \Omega, M}^{(\alpha, \mu)}+\left|\rho_{1}\right|_{s+1, \Gamma, M}^{(3+\alpha)}+\left|\rho_{2}\right|_{s+1, \Sigma, M}^{(3+\alpha, \mu)} \\
& \leq C\left(|\mathbf{f}|_{s-2, \Omega, M}^{(\alpha, \mu)}+|g|_{s-1, \Omega, M}^{(1+\alpha, \mu)}+|\mathbf{a}|_{s-1, \Gamma, M}^{(1+\alpha)}+\left|a_{3}\right|_{s, \Gamma, M}^{(2+\alpha)}+|\mathbf{b}|_{s-1, \Sigma, M}^{(1+\alpha, \mu)}\right. \\
&\left.\quad+\left|b_{3}\right|_{s, \Sigma, M}^{(2+\alpha, \mu)}+\left|h_{1}\right|_{s-1, \Gamma, M}^{(1+\alpha)}+\left|h_{2}\right|_{s-1, \Sigma, M}^{(1+\alpha, \mu)}+\left|m_{1}\right|_{M}^{(s)}+\left|m_{2}\right|_{M}^{(s)}\right)
\end{aligned}
$$

for some constant $C>0$ that is independent of the data.

We begin with the following lemma.

Lemma 4 Under the assumption stated in Theorem 2 for $g, a_{3}$, and $b_{3}$, there exists a vector field $\mathbf{w} \in C_{s}^{2+\alpha, \mu}(\Omega, M)$ satisfying

$$
\left\{\begin{array}{l}
\nabla \cdot \mathbf{w}=g \quad \text { in } \Omega, \\
\mathbf{w} \cdot \mathbf{n}=a_{3} \quad \text { on } \Gamma, \quad \mathbf{w} \cdot \mathbf{n}=b_{3} \quad \text { on } \Sigma
\end{array}\right.
$$

and the estimate

$$
|\mathbf{w}|_{s, \Omega, M}^{(2+\alpha, \mu)} \leq C\left(|g|_{s-1, \Omega, M}^{(1+\alpha, \mu)}+\left|a_{3}\right|_{s, \Gamma, M}^{(2+\alpha)}+\left|b_{3}\right|_{s, \Sigma, M}^{(2+\alpha, \mu)}\right)
$$

for a constant $C>0$ that is independent of the data. 
Proof We construct the function in the form $\mathbf{w}=\nabla \Phi$, where $\Phi$ is a solution of

$$
\left\{\begin{array}{l}
\Delta \Phi=g \quad \text { in } \Omega, \\
\left.\frac{\partial \Phi}{\partial n}\right|_{\Gamma}=a_{3},\left.\quad \frac{\partial \Phi}{\partial n}\right|_{\Sigma}=b_{3} .
\end{array}\right.
$$

We first construct a function $\Phi^{\prime}$ satisfying conditions $(38)^{2,3}$ and the estimate

$$
\left|\Phi^{\prime}\right|_{s+1, \Omega, M}^{(3+\alpha, \mu)} \leq C\left(\left|a_{3}\right|_{s, \Gamma, M}^{(2+\alpha)}+\left|b_{3}\right|_{s, \Sigma, M}^{(2+\alpha, \mu)}\right) .
$$

Then we introduce the new unknown $\Phi-\Phi^{\prime}$ to reduce problem (38) to one in which $a_{3}=b_{3}=0$. In the following argument, we again denote $\Phi-\Phi^{\prime}$ and $g-\Delta \Phi^{\prime}$ by $\Phi$ and $g$, respectively.

Now, let us set

$$
D_{2}^{1}(\Omega)=\left\{u \in L_{2, \text { loc }}(\Omega) \mid \partial_{x} u \in L_{2}(\Omega)\right\}
$$

For $u \in D_{2}^{1}(\Omega)$, we set

$$
[u]=\left\{w \in D_{2}^{1}(\Omega) \mid w=u+c \text { for a constant } c\right\},
$$

and let $\dot{D}_{2}^{1}(\Omega)$ be the space of all equivalence classes $[u]$. As shown in [6], the space $\dot{D}_{2}^{1}(\Omega)$ is a Hilbert space with the scalar product

$$
(u, v)=\int_{\Omega} \nabla u \cdot \nabla v d x
$$

We prove the weak solvability of problem (38) with $a_{3}=b_{3}=0$ in class $\dot{D}_{2}^{1}(\Omega)$ for arbitrary $g \in L_{2}(\Omega)$. Let $\zeta_{k}(k \in \mathbb{N})$ be the cut-off function such that

$$
\zeta_{k}(x)= \begin{cases}1 & \left(x_{3} \geq-k\right) \\ 0 & \left(x_{3} \leq-(k+1)\right) .\end{cases}
$$

Set $g_{k}=\zeta_{k} g$ and let $\Phi_{k}$ be the corresponding solution of the problem with $g=g_{k}$. We multiply the equation $\Delta \Phi_{k}=g_{k}$ by $\phi \in \dot{D}_{2}^{1}(\Omega)$ and integrate both sides to obtain

$$
-\int_{\Omega} \nabla \Phi_{k} \cdot \nabla \phi d x=\int_{\Omega} g_{k} \phi d x
$$

The Riesz representation theorem then implies that there exists a unique solution $\Phi_{k} \in$ $\dot{D}_{2}^{1}(\Omega)$ of (39), and we obtain the estimate

$$
\left\|\nabla \Phi_{k}\right\|_{2, \Omega} \leq C\left\|g_{k}\right\|_{2, \Omega}
$$

Thus, by taking the limit $k \rightarrow \infty$ in (39), we have a solution $\Phi \in \dot{D}_{2}^{1}(\Omega)$ satisfying the estimate

$$
\|\nabla \Phi\|_{2, \Omega} \leq C\|g\|_{2, \Omega} .
$$


For problem (38) with $a_{3}=b_{3}=0$, we can obtain a decay estimate that is similar to (56). Based on this estimate, we can obtain estimate (37) using a method of localization similar to that used to obtain estimates (71) and (73). Because of the axial symmetry of the data and the domain, the solution constructed above is axially symmetric. Therefore, we can perform the above localization procedure for a two-dimensional problem using estimate (41) given below. In Lemma $5, d_{\theta}, \gamma_{\theta}, \gamma_{0}$, and $M$ are defined in the manner specified in Sect. 4. As the result is well known, the proof is omitted.

Lemma 5 Assume that $0<\alpha<1, s \notin \mathbb{Z}, 0<s<\pi / \theta$, and $s \leq 2+\alpha$. Further, assume that $g \in$ $C_{s-1}^{1+\alpha}\left(d_{\theta}, M\right), a_{3} \in C_{s}^{2+\alpha}\left(\gamma_{\theta}, M\right)$, and $b_{3} \in C_{s}^{2+\alpha}\left(\gamma_{0}, M\right)$, and that their supports are compact. Additionally, assume that the condition

$$
\int_{d_{\theta}} g d x=\int_{\gamma_{\theta}} a_{3} d S+\int_{\gamma_{0}} b_{3} d S
$$

is satisfied. Then problem (38) with $\Omega=d_{\theta}, \Gamma=\gamma_{\theta}$, and $\Sigma=\gamma_{0}$ has a unique solution $\nabla \Phi \in$ $C_{s}^{2+\alpha}\left(d_{\theta}, M\right)$ that satisfies the estimate

$$
|\nabla \Phi|_{s, d_{\theta}, M}^{(2+\alpha)} \leq C\left(|g|_{s-1, \gamma_{\theta}, M}^{(1+\alpha)}+\left|a_{3}\right|_{s, \gamma_{\theta}, M}^{(2+\alpha)}+\left|b_{3}\right|_{s, \gamma_{0}, M}^{(2+\alpha)}\right)
$$

for a constant $C>0$ that is independent of the data.

Thus, we have proved Lemma 4.

Lemma 6 Under the assumptions stated in Theorem 2 for $h_{1}, h_{2}, m_{1}$, and $m_{2}$, there exists a unique solution $\left(s_{1}, s_{2}\right)$ to the problem

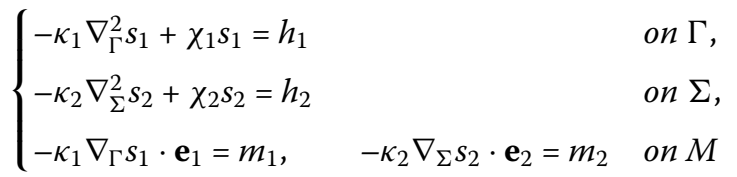

satisfying the estimate

$$
\begin{aligned}
& \left|s_{1}\right|_{s+1, \Gamma, M}^{(3+\alpha)}+\left|s_{2}\right|_{s+1, \Sigma, M}^{(3+\alpha, \mu)} \\
& \quad \leq C\left(\left|h_{1}\right|_{s-1, \Gamma, M}^{(1+\alpha)}+\left|h_{2}\right|_{s-1, \Sigma, M}^{(1+\alpha, \mu)}+\left|m_{1}\right|_{M}^{(s)}+\left|m_{2}\right|_{M}^{(s)}\right)
\end{aligned}
$$

for a constant $C>0$ that is independent of the data.

Proof The proof is similar to that of Lemma 4. The existence of a weak solution of problem (42) is easily shown. In addition, for the problem

$$
\begin{cases}-\Delta \rho+\chi \rho=h & \text { in } \mathbb{R}_{+}^{2} \equiv\left\{\left(z_{1}, z_{2}\right) \in \mathbb{R}^{2} \mid z_{2}>0\right\} \\ \left.\partial_{z_{2}} \rho\right|_{z_{2}=0}=H, & \rho \rightarrow 0\left(z_{2} \rightarrow \infty\right),\end{cases}
$$

where $\chi \geq 0$ is a constant, we can obtain the following estimate in the same manner as in the proof of Theorem 7.1 in [30] under the assumption that the supports of the data are 
compact:

$$
|\rho|_{s+1, \mathbb{R}_{+}^{2}, \mathbb{R}}^{(3+\alpha)} \leq C\left(|h|_{s-1, \mathbb{R}_{+}^{2}, \mathbb{R}}^{(1+\alpha)}+|H|_{\mathbb{R}}^{(s)}\right) .
$$

Using this estimate, in addition to a decay estimate for $s_{1}, s_{2}$ similar to that in (56), we achieve the desired result in a similar manner to that used to obtain estimates (71) and (73).

With the aid of Lemmas 4 and 6 , problem (32) reduces to the problem with $g=a_{3}=b_{3}=$ $h_{1}=h_{2}=m_{1}=m_{2}=0$. Therefore, we hereafter consider (32) under this assumption.

Let us prove the weak solvability of the problem. After multiplying $(32)^{1}$ by $\mathbf{v}^{\prime},(32)^{5}$ by $\rho_{1}^{\prime}$, and $(32)^{8}$ by $\rho_{2}^{\prime}$, we use integration by parts to obtain

$$
\begin{aligned}
& 2 v \int_{\Omega} \mathbf{D}(\mathbf{v}): \mathbf{D}\left(\mathbf{v}^{\prime}\right) d x+\beta \int_{\Sigma} \mathbf{v} \cdot \mathbf{v}^{\prime} d S+\kappa_{1} \gamma \int_{\Gamma} \nabla_{\Gamma} \rho_{1} \cdot \nabla_{\Gamma} \rho_{1}^{\prime} d S \\
& \quad+\chi_{1} \gamma \int_{\Gamma} \rho_{1} \rho_{1}^{\prime} d S+\kappa_{2} \gamma \int_{\Sigma} \nabla_{\Sigma} \rho_{2} \cdot \nabla_{\Sigma} \rho_{2}^{\prime} d S+\chi_{2} \gamma \int_{\Sigma} \rho_{2} \rho_{2}^{\prime} d S \\
& \quad+\gamma \int_{\Gamma}\left(\nabla_{\Gamma} \rho_{1} \cdot \mathbf{v}^{\prime}-\nabla_{\Gamma} \rho_{1}^{\prime} \cdot \mathbf{v}\right) d S+\frac{\gamma}{2} \int_{\Sigma}\left(\nabla_{\Sigma} \rho_{2} \cdot \mathbf{v}^{\prime}-\nabla_{\Sigma} \rho_{2}^{\prime} \cdot \mathbf{v}\right) d S \\
& =\int_{\Omega} \mathbf{f} \cdot \mathbf{v}^{\prime} d x+\int_{\Gamma} \mathbf{a} \cdot \mathbf{v}^{\prime} d S+\int_{\Sigma} \mathbf{b} \cdot \mathbf{v}^{\prime} d S .
\end{aligned}
$$

Let $J(\Omega)$ be the function space defined by

$$
J(\Omega) \equiv\left\{\mathbf{f} \in W_{2}^{1}(\Omega)|\nabla \cdot \mathbf{f}=0, \mathbf{f} \cdot \mathbf{n}|_{\Gamma \cup \Sigma}=0\right\} .
$$

We now prove the following inequality. The main difficulty is in the estimation of $\|\mathbf{v}\|_{2, \Omega}$. A similar inequality in an infinite strip domain is derived in [18]. Because the domain is three-dimensional in the present case, more complicated arguments are necessary.

Lemma 7 For arbitrary $\mathbf{v} \in J(\Omega)$, the following inequality holds:

$$
\left(\|\mathbf{v}\|_{2, \Omega}^{(1)}\right)^{2} \leq C \int_{\Omega} \mathbf{D}(\mathbf{v}): \mathbf{D}(\mathbf{v}) d x
$$

Proof For any $\mathbf{v} \in J(\Omega)$, with the aid of Korn's inequality, we have

$$
\left\|\partial_{x} \mathbf{v}\right\|_{2, \Omega}^{2} \leq C\left(\int_{\Omega} \mathbf{D}(\mathbf{v}): \mathbf{D}(\mathbf{v}) d x+\|\mathbf{v}\|_{2, \Gamma}^{2}\right)
$$

As $\Gamma$ is compact, using a similar argument as in the proof of Lemma 4 in [33] implies that there exists a constant $C(\epsilon)$ satisfying the inequality

$$
\|\mathbf{v}\|_{2, \Gamma}^{2} \leq \epsilon\left\|\partial_{x} \mathbf{v}\right\|_{2, \Omega}^{2}+C(\epsilon) \int_{\Omega} \mathbf{D}(\mathbf{v}): \mathbf{D}(\mathbf{v}) d x
$$

for arbitrary $\epsilon>0$. Combining these inequalities, we have

$$
\left\|\partial_{x} \mathbf{v}\right\|_{2, \Omega}^{2} \leq C \int_{\Omega} \mathbf{D}(\mathbf{v}): \mathbf{D}(\mathbf{v}) d x .
$$


Now, from the conditions $\nabla \cdot \mathbf{v}=0$ and $\left.\mathbf{v} \cdot \mathbf{n}\right|_{\Sigma}=0, \int_{B} v_{3}\left(x^{\prime}, x_{3}\right) d x^{\prime}=0$ holds for arbitrary $x_{3}<0$. From this, we have

$$
\left\|v_{3}\left(\cdot, x_{3}\right)\right\|_{2, B} \leq C\left\|\nabla_{x^{\prime}} v_{3}\left(\cdot, x_{3}\right)\right\|_{2, B}
$$

By integrating the identity with $n=2$,

$$
\sum_{i, j=1}^{n}\left\{\partial_{x_{i}}\left(v_{i} x_{j} v_{j}\right)-\left(\partial_{x_{i}} v_{i}\right) x_{j} v_{j}-v_{i} x_{j}\left(\partial_{x_{i}} v_{j}\right)\right\}-|\mathbf{v}|^{2}=0
$$

on $B$ under the condition that $\left.\mathbf{v} \cdot \mathbf{n}\right|_{\partial B}=0$, we obtain

$$
\left\|\mathbf{v}^{\prime}\left(\cdot, x_{3}\right)\right\|_{2, B} \leq C\left\|\partial_{x^{\prime}} \mathbf{v}^{\prime}\left(\cdot, x_{3}\right)\right\|_{2, B},
$$

where $\mathbf{v}^{\prime}=\left(v_{1}, v_{2}\right)$. We integrate (48) and (50) with respect to $x_{3}$ to obtain

$$
\|\mathbf{v}\|_{2, \Omega_{1}} \leq C\left\|\partial_{x} \mathbf{v}\right\|_{2, \Omega_{1}}
$$

Next, we integrate (49) with $n=3$ in the domain $\Omega_{0,1}$ and find that

$$
\|\mathbf{v}\|_{2, \Omega_{0,1}} \leq C\left(\left\|\partial_{x} \mathbf{v}\right\|_{2, \Omega_{0,1}}+\|\mathbf{v}(\cdot,-1)\|_{2, B}\right),
$$

and we note the estimate

$$
\|\mathbf{v}(\cdot,-1)\|_{2, B} \leq C\|\mathbf{v}\|_{2, \Omega_{1}}^{(1)}
$$

from (51) and (52) to obtain

$$
\|\mathbf{v}\|_{2, \Omega} \leq C\left\|\partial_{x} \mathbf{v}\right\|_{2, \Omega}
$$

Estimate (46) is obtained by combining inequalities (47) and (53).

We now introduce the Lax-Milgram theorem.

Theorem 3 (Lax-Milgram [14]) Let $H$ be a real Hilbert space with the norm $\|\cdot\|$ and $B: H \times H \rightarrow \mathbb{R}$ be a bilinear mapping. If there exist constants $\alpha, \beta>0$ such that

$$
\begin{aligned}
& |B[u, v]| \leq \alpha\|u\|\|v\| \quad(u, v \in H), \\
& \beta\|u\|^{2} \leq B[u, u] \quad(u \in H) .
\end{aligned}
$$

Then, for every linear functional $f: H \rightarrow \mathbb{R}$, there exists a unique element $u \in H$ such that $B[u, v]=f(v)$ for all $v \in H$.

Lemma 7 indicates that the bilinear form defined by the left-hand side of (45), which we denote as $B\left[\left(\mathbf{v}, \rho_{1}, \rho_{2}\right),\left(\mathbf{v}^{\prime}, \rho_{1}^{\prime}, \rho_{2}^{\prime}\right)\right]$, satisfies the condition

$$
B\left[\left(\mathbf{v}, \rho_{1}, \rho_{2}\right),\left(\mathbf{v}, \rho_{1}, \rho_{2}\right)\right] \geq C\left\{\left(\|\mathbf{v}\|_{2, \Omega}^{(1)}\right)^{2}+\left(\left\|\rho_{1}\right\|_{2, \Gamma}^{(1)}\right)^{2}+\left(\left\|\rho_{2}\right\|_{2, \Sigma}^{(1)}\right)^{2}\right\} .
$$

Thus, from Theorem 3, we have the following. 
Theorem 4 For arbitrary $(\mathbf{f}, \mathbf{a}, \mathbf{b}) \in L_{2}(\Omega) \times L_{2}(\Gamma) \times L_{2}(\Sigma)$, there exists a unique solution $\left(\mathbf{v}, \rho_{1}, \rho_{2}\right) \in J(\Omega) \times W_{2}^{1}(\Gamma) \times W_{2}^{1}(\Sigma)$ satisfying $(45)$ for all $\left(\mathbf{v}^{\prime}, \rho_{1}^{\prime}, \rho_{2}^{\prime}\right) \in J(\Omega) \times W_{2}^{1}(\Gamma) \times$ $W_{2}^{1}(\Sigma)$ and the estimate

$$
\|\mathbf{v}\|_{2, \Omega}^{(1)}+\left\|\rho_{1}\right\|_{2, \Gamma}^{(1)}+\left\|\rho_{2}\right\|_{2, \Sigma}^{(1)} \leq C\left(\|\mathbf{f}\|_{2, \Omega}+\|\mathbf{a}\|_{2, \Gamma}+\|\mathbf{b}\|_{2, \Sigma}\right)
$$

for a constant $C>0$ that is independent of the data.

For the solution $\left(\mathbf{v}, \rho_{1}, \rho_{2}\right)$ obtained above, the pressure $p$ is determined from the following equation defined for arbitrary $\eta \in H(\Omega) \equiv\left\{\mathbf{f} \in W_{2}^{1}(\Omega)|\mathbf{f} \cdot \mathbf{n}|_{\Gamma \cup \Sigma}=0\right\}$ with a compact support:

$$
\begin{aligned}
2 v & \int_{\Omega} \mathbf{D}(\mathbf{v}): \mathbf{D}(\eta) d x+\beta \int_{\Sigma} \mathbf{v} \cdot \eta d S-\int_{\Omega} p \nabla \cdot \eta d x \\
& =\int_{\Omega} \mathbf{f} \cdot \eta d x+\int_{\Gamma}\left(\mathbf{a}-\gamma \nabla_{\Gamma} \rho_{1}\right) \cdot \eta d S+\int_{\Sigma}\left(\mathbf{b}-\frac{\gamma}{2} \nabla_{\Sigma} \rho_{2}\right) \cdot \eta d S .
\end{aligned}
$$

If we take the vector $\eta$ such that

$$
\nabla \cdot \eta=p \quad \text { in } \Omega^{\prime}, \quad \eta=\mathbf{0} \quad \text { in } \Omega \backslash \Omega^{\prime}, \quad\left\|\partial_{x} \eta\right\|_{2, \Omega} \leq C\|p\|_{2, \Omega^{\prime}},
$$

where $\Omega^{\prime} \subset \Omega$ is a bounded domain (for the construction of $\eta$, see, e.g., [13]), we have the estimate

$$
\|p\|_{2, \Omega^{\prime}} \leq C\left(\|\mathbf{f}\|_{2, \Omega}+\|\mathbf{a}\|_{2, \Gamma}+\|\mathbf{b}\|_{2, \Sigma}\right)
$$

Here, $p$ is normalized by the condition $\int_{\Omega^{\prime}} p d x=0$.

The axial symmetry of the solution constructed above readily follows from the symmetry of the data and the domain. In addition, when $f_{\phi}=a_{\phi}=b_{\phi}=0$, if we take $v_{\phi} \mathbf{e}_{\phi}$ $\left(\mathbf{e}_{\phi}=\left(-x_{2} /\left|x^{\prime}\right|, x_{1} /\left|x^{\prime}\right|, 0\right)\right)$ as $\mathbf{v}^{\prime}$ in (45) and take 0 as $\rho_{1}^{\prime}$ and $\rho_{2}^{\prime}$, we have the equality

$$
\frac{v}{2} \int_{\Omega}\left(\frac{\partial v_{\phi}}{\partial z}\right)^{2}+\left(\frac{\partial v_{\phi}}{\partial r}-\frac{v_{\phi}}{r}\right)^{2} r d r d \phi d z+\beta \int_{\Sigma} v_{\phi}^{2} d \phi d z=0
$$

which indicates that $v_{\phi}=0$.

The following decay estimate is obtained in a similar manner to the proofs of Theorem 5.3 in [27] and Lemma 1 in [19].

Lemma 8 Let $\Omega(\lambda, t)=\left\{x \in \Omega \mid \lambda-t<x_{3}<\lambda+t\right\}$ and $\Sigma(\lambda, t)=\left\{x \in \Sigma \mid \lambda-t<x_{3}<\lambda+t\right\}(\lambda<$ $-4)$. Assume that $\mathbf{f}, \mathbf{a}$, and $\mathbf{b}$ possess the same regularity assumed in Theorem 2 . Then the solutions $\mathbf{v}$ and $\rho_{2}$ of (45) satisfy the inequality

$$
\|\mathbf{v}\|_{2, \Omega(\lambda, 1)}^{(1)}+\left\|\rho_{2}\right\|_{2, \Sigma(\lambda, 1)}^{(1)} \leq C e^{\mu \lambda}\left(|\mathbf{f}|_{s-2, \Omega, M}^{(\alpha, \mu)}+|\mathbf{a}|_{s-1, \Gamma, M}^{(1+\alpha)}+|\mathbf{b}|_{s-1, \Sigma, M}^{(1+\alpha, \mu)}\right)
$$

for constants $\mu>0$ and $C>0$ that are independent of the data. 
Proof We use integration by parts in the domain $\Omega(\lambda, t)$ in a manner similar to that for (45) to obtain

$$
\begin{aligned}
& 2 v \int_{\Omega(\lambda, t)} \mathbf{D}(\mathbf{v}): \mathbf{D}(\mathbf{v}) d x+\beta \int_{\Sigma(\lambda, t)}|\mathbf{v}|^{2} d S \\
& \quad+\gamma \kappa_{2} \int_{\Sigma(\lambda, t)}\left|\nabla_{\Sigma} \rho_{2}\right|^{2} d S+\gamma \chi_{2} \int_{\Sigma(\lambda, t)} \rho_{2}^{2} d S \\
& \quad+\gamma \int_{\partial \omega(\lambda+t)} \rho_{2}\left(\frac{\mathbf{v}}{2}-\kappa_{2} \nabla_{\Sigma} \rho_{2}\right) \cdot \mathbf{e}_{3} d l-\gamma \int_{\partial \omega(\lambda-t)} \rho_{2}\left(\frac{\mathbf{v}}{2}-\kappa_{2} \nabla_{\Sigma} \rho_{2}\right) \cdot \mathbf{e}_{3} d l \\
& \quad-\int_{\omega(\lambda+t)} \mathbf{T}(\mathbf{v}, p) \mathbf{e}_{3} \cdot \mathbf{v} d x^{\prime}+\int_{\omega(\lambda-t)} \mathbf{T}(\mathbf{v}, p) \mathbf{e}_{3} \cdot \mathbf{v} d x^{\prime} \\
& =\int_{\Omega(\lambda, t)} \mathbf{f} \cdot \mathbf{v} d x+\int_{\Sigma(\lambda, t)} \mathbf{b} \cdot \mathbf{v} d S,
\end{aligned}
$$

where $\mathbf{e}_{3}=(0,0,1)$ and $\omega(t)=\left\{x_{3}=t, x^{\prime} \in B\right\}$.

We integrate (57) with respect to $t$ over the interval $(\eta-1, \eta)(1<\eta)$ to obtain

$$
\begin{aligned}
Z(\lambda, \eta) \equiv & \int_{\eta-1}^{\eta}\left(\|\mathbf{v}\|_{2, \Omega(\lambda, t)}^{(1)}\right)^{2}+\left(\left\|\rho_{2}\right\|_{2, \Sigma(\lambda, t)}^{(1)}\right)^{2} d t \\
\leq & C\left\{\left|\int_{\tilde{D}(\lambda, \eta)} \mathbf{T}(\mathbf{v}, p) \mathbf{e}_{3} \cdot \mathbf{v} d x\right|+\left|\int_{D(\lambda, \eta)} \mathbf{T}(\mathbf{v}, p) \mathbf{e}_{3} \cdot \mathbf{v} d x\right|\right. \\
& +\left|\int_{\tilde{S}(\lambda, \eta)} \rho_{2}\left(\frac{1}{2} \mathbf{v}-\nabla_{\Sigma} \rho_{2}\right) \cdot \mathbf{e}_{3} d S\right|+\left|\int_{S(\lambda, \eta)} \rho_{2}\left(\frac{1}{2} \mathbf{v}-\nabla_{\Sigma} \rho_{2}\right) \cdot \mathbf{e}_{3} d S\right| \\
& \left.+\left|\int_{\eta-1}^{\eta}\left(\int_{\Omega(\lambda, t)} \mathbf{f} \cdot \mathbf{v} d x\right) d t\right|+\left|\int_{\eta-1}^{\eta}\left(\int_{\Sigma(\lambda, t)} \mathbf{b} \cdot \mathbf{v} d S\right) d t\right|\right\},
\end{aligned}
$$

where

$$
\left\{\begin{array}{l}
\tilde{D}(\lambda, \eta)=\left\{x \in \Omega \mid \lambda+\eta-1<x_{3}<\lambda+\eta\right\} \\
D(\lambda, \eta)=\left\{x \in \Omega \mid \lambda-\eta<x_{3}<\lambda-\eta+1\right\} \\
\tilde{S}(\lambda, \eta)=\left\{x \in \Sigma \mid \lambda+\eta-1<x_{3}<\lambda+\eta\right\} \\
S(\lambda, \eta)=\left\{x \in \Sigma \mid \lambda-\eta<x_{3}<\lambda-\eta+1\right\} .
\end{array}\right.
$$

The terms on the right-hand side are estimated as follows:

$$
\begin{aligned}
& \left|\int_{\tilde{D}(\lambda, \eta)} \mathbf{T}(\mathbf{v}, p) \mathbf{e}_{3} \cdot \mathbf{v} d x\right| \leq C\left\{\left(\|\mathbf{v}\|_{2, \tilde{D}(\lambda, \eta)}^{(1)}\right)^{2}+\|\mathbf{f}\|_{2, \tilde{D}(\lambda, \eta)}^{2}\right\}, \\
& \left|\int_{D(\lambda, \eta)} \mathbf{T}(\mathbf{v}, p) \mathbf{e}_{3} \cdot \mathbf{v} d x\right| \leq C\left\{\left(\|\mathbf{v}\|_{2, D(\lambda, \eta)}^{(1)}\right)^{2}+\|\mathbf{f}\|_{2, D(\lambda, \eta)}^{2}\right\}, \\
& \left|\int_{\tilde{S}(\lambda, \eta)} \rho_{2}\left(\frac{1}{2} \mathbf{v}-\nabla_{\Sigma} \rho_{2}\right) \cdot \mathbf{e}_{3} d S\right| \leq C\left\{\left(\|\mathbf{v}\|_{2, \tilde{D}(\lambda, \eta)}^{(1)}\right)^{2}+\left(\left\|\rho_{2}\right\|_{2, \tilde{S}(\lambda, \eta)}^{(1)}\right)^{2}\right\}, \\
& \left|\int_{S(\lambda, \eta)} \rho_{2}\left(\frac{1}{2} \mathbf{v}-\nabla_{\Sigma} \rho_{2}\right) \cdot \mathbf{e}_{3} d S\right| \leq C\left\{\left(\|\mathbf{v}\|_{2, D(\lambda, \eta)}^{(1)}\right)^{2}+\left(\left\|\rho_{2}\right\|_{2, S(\lambda, \eta)}^{(1)}\right)^{2}\right\},
\end{aligned}
$$


and

$$
\begin{aligned}
& \left|\int_{\eta-1}^{\eta}\left(\int_{\Omega(\lambda, t)} \mathbf{f} \cdot \mathbf{v} d x\right) d t\right|+\left|\int_{\eta-1}^{\eta}\left(\int_{\Sigma(\lambda, t)} \mathbf{b} \cdot \mathbf{v} d S\right) d t\right| \\
& \leq \epsilon Z(\lambda, \eta)+C(\epsilon)\left(\|\mathbf{f}\|_{2, \Omega(\lambda, \eta)}^{2}+\|\mathbf{b}\|_{2, \Sigma(\lambda, \eta)}^{2}\right)
\end{aligned}
$$

where $\epsilon>0$ is an arbitrary constant.

Thus, from (58), we have

$$
\begin{aligned}
Z(\lambda, \eta) \leq & C_{1}\left\{\left(\|\mathbf{v}\|_{2, \tilde{D}(\lambda, \eta)}^{(1)}\right)^{2}+\left(\|\mathbf{v}\|_{2, D(\lambda, \eta)}^{(1)}\right)^{2}\right. \\
& \left.+\left(\left\|\rho_{2}\right\|_{2, \tilde{S}(\lambda, \eta)}^{(1)}\right)^{2}+\left(\left\|\rho_{2}\right\|_{2, S(\lambda, \eta)}^{(1)}\right)^{2}\right\}+C_{2}\left(\|\mathbf{f}\|_{2, \Omega(\lambda, \eta)}^{2}+\|\mathbf{b}\|_{2, \Sigma(\lambda, \eta)}^{2}\right) \\
= & C_{1} \frac{d}{d \eta} Z(\lambda, \eta)+C_{2}\left(\|\mathbf{f}\|_{2, \Omega(\lambda, \eta)}^{2}+\|\mathbf{b}\|_{2, \Sigma(\lambda, \eta)}^{2}\right) .
\end{aligned}
$$

Multiplying this inequality by $e^{-\eta / c_{1}}$ and integrating over the interval $(2,-\lambda / 2)$ with respect to $\eta$ gives

$Z(\lambda, 2)$

$$
\leq Z\left(\lambda,-\frac{\lambda}{2}\right) e^{\frac{\lambda+4}{2 C_{1}}}+\frac{C_{2}}{C_{1}} \int_{2}^{-\frac{\lambda}{2}}\left(\|\mathbf{f}\|_{2, \Omega(\lambda, \eta)}^{2}+\|\mathbf{b}\|_{2, \Sigma(\lambda, \eta)}^{2}\right) e^{-\frac{\eta-2}{C_{1}}} d \eta .
$$

Now, we note the following inequalities:

$$
\begin{aligned}
\left(\|\mathbf{v}\|_{2, \Omega(\lambda, 1)}^{(1)}\right)^{2}+\left(\left\|\rho_{2}\right\|_{2, \Sigma(\lambda, 1)}^{(1)}\right)^{2} \leq Z(\lambda, 2), & \\
Z\left(\lambda,-\frac{\lambda}{2}\right) & \leq\left(\|\mathbf{v}\|_{2, \Omega}^{(1)}\right)^{2}+\left(\left\|\rho_{2}\right\|_{2, \Sigma}^{(1)}\right)^{2} \\
& \leq C\left\{\left(|\mathbf{f}|_{s-2, \Omega, M}^{(\alpha, \mu)}\right)^{2}+\left(|\mathbf{a}|_{s-1, \Gamma, M}^{(1+\alpha)}\right)^{2}+\left(|\mathbf{b}|_{s-1, \Sigma, M}^{(1+\alpha, \mu)}\right)^{2}\right\},
\end{aligned}
$$

and

$$
\begin{aligned}
& \int_{2}^{-\frac{\lambda}{2}}\left(\|\mathbf{f}\|_{2, \Omega(\lambda, \eta)}^{2}+\|\mathbf{b}\|_{2, \Sigma(\lambda, \eta)}^{2}\right) e^{-\frac{\eta-2}{C_{1}}} d \eta \\
& \quad \leq C e^{2 \mu \lambda}\left\{\left(|\mathbf{f}|_{s-2, \Omega, M}^{(\alpha, \mu)}\right)^{2}+\left(|\mathbf{b}|_{s-1, \Sigma, M}^{(1+\alpha, \mu)}\right)^{2}\right\}
\end{aligned}
$$

to obtain the desired estimate (56) for $0<\mu<1 / 2 C_{1}$.

Let us proceed to the estimation of the higher-order derivatives of the solution through Schauder's method. We introduce a covering $\left\{U_{i}\right\}_{i \in \mathbb{N}}$ of $\bar{\Omega}$, where $\left\{U_{i}\right\}$ is a family of balls with the following properties:

(i) if $U_{i} \cap M \neq \phi$, then $\xi_{i}$, the center of $U_{i}$, is on $M$; if $U_{i} \cap M=\phi$ and $U_{i} \cap \partial \Omega \neq \phi$, then $\xi_{i}$ is on $\Gamma$ or $\Sigma$;

(ii) the radius of $U_{i}\left(U_{i} \cap \partial \Omega \neq \phi\right)$ is sufficiently small that $U_{i}$ divides the surface $\partial \Omega$ into two connected parts; and 
(iii) there exists an integer $N_{0}$ such that the intersection of $N_{0}+1$ arbitrary different balls is empty.

We further introduce a partition of unity $\left\{\zeta_{i}\right\}$ corresponding to the covering $\left\{U_{i}\right\}$.

For $U_{i}$ with $\xi_{i} \notin M$, the following estimates are obtained (for the derivation, see [11]):

$$
\begin{aligned}
& |\mathbf{v}|_{\Omega_{i}^{\prime}}^{(2+\alpha)}+|\nabla p|_{\Omega_{i}^{\prime}}^{(\alpha)} \leq C\left(|\mathbf{f}|_{\Omega_{i}}^{(\alpha)}+\|\mathbf{v}\|_{2, \Omega_{i}}\right) \quad\left(\xi_{i} \in \Omega\right), \\
& |\mathbf{v}|_{\Omega_{i}^{\prime}}^{(2+\alpha)}+|\nabla p|_{\Omega_{i}^{\prime}}^{(\alpha)}+\left|\rho_{1}\right|_{\Gamma_{i}^{\prime}}^{(3+\alpha)} \\
& \quad \leq C\left(|\mathbf{f}|_{\Omega_{i}}^{(\alpha)}+|\mathbf{a}|_{\Gamma_{i}}^{(1+\alpha)}+\|\mathbf{v}\|_{2, \Omega_{i}}+\left\|\rho_{1}\right\|_{2, \Gamma_{i}}\right) \quad\left(\xi_{i} \in \Gamma\right), \\
& |\mathbf{v}|_{\Omega_{i}^{\prime}}^{(2+\alpha)}+|\nabla p|_{\Omega_{i}^{\prime}}^{(\alpha)}+\left|\rho_{2}\right|_{\Sigma_{i}^{\prime}}^{(3+\alpha)} \\
& \quad \leq C\left(|\mathbf{f}|_{\Omega_{i}}^{(\alpha)}+|\mathbf{b}|_{\Sigma_{i}}^{(1+\alpha)}+\|\mathbf{v}\|_{2, \Omega_{i}}+\left\|\rho_{2}\right\|_{2, \Sigma_{i}}\right) \quad\left(\xi_{i} \in \Sigma\right),
\end{aligned}
$$

where $D_{i}$ and $D_{i}^{\prime}$ denote the domains $D \cap U_{i}$ and $D \cap U_{i}^{\prime}$, respectively, for $D=\Omega, \Gamma$, $\Sigma$, and $U_{i}^{\prime}$ denotes a subset of $U_{i}$ on which $\zeta_{i}=1$ holds. To estimate the solution defined on $U_{i}$ with $\xi_{i} \in M$, we use the axial symmetry of the solution and rewrite problem (32) as the following two-dimensional problem in cylindrical coordinates:

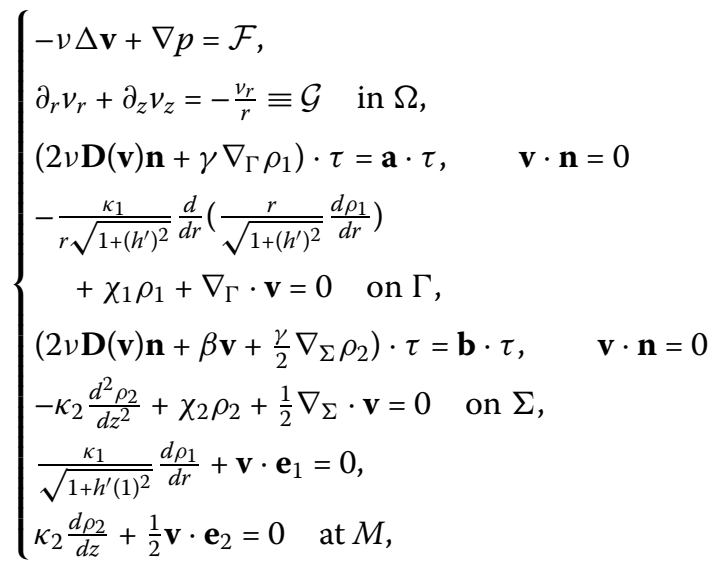

where

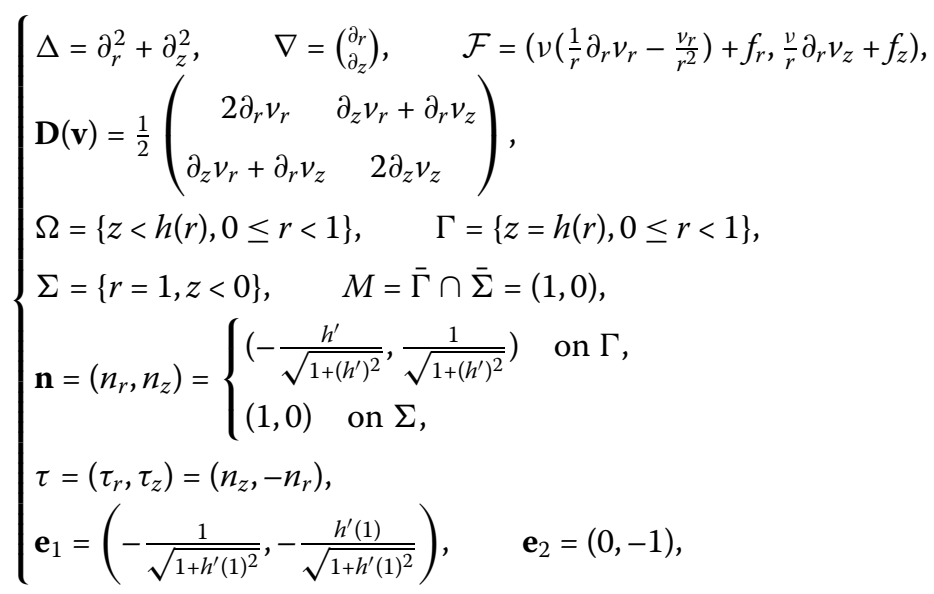

and $f_{r}$ and $f_{z}$ denote the $r$-component and $z$-component, respectively, of the term $\mathbf{f}$. 
We rewrite the above problem in the coordinates $\left(y_{1}, y_{2}\right)$, which are related to the original coordinates by $\left(\begin{array}{c}y_{1} \\ y_{2}\end{array}\right)=\mathcal{R}\left(\begin{array}{c}r-1 \\ z\end{array}\right)$, where $\mathcal{R}$ is the matrix of rotation $\theta-(3 / 2) \pi$ around the origin. In the coordinates $\{y\}, \Sigma$ is given by the line $y_{2}=y_{1} \tan \theta$ (or $y_{1}=0$ when $\theta=\pi / 2$ ), and we represent $\Gamma$ as the curve $y_{2}=g\left(y_{1}\right)$.

We now introduce a mapping $y=\Phi(\eta)=\left(\eta_{1}, \eta_{2}\right)+\left(\Phi_{1}(\eta), \Phi_{2}(\eta)\right)$, where $\Phi_{1}$ and $\Phi_{2}$ are constructed to satisfy the following conditions:

$$
\left.\left(\Phi_{1}(\eta), \Phi_{2}(\eta)\right)\right|_{\gamma_{0}}=\left(0, \tilde{g}\left(\eta_{1}\right)\right),\left.\quad\left(\Phi_{1}(\eta), \Phi_{2}(\eta)\right)\right|_{\gamma_{\theta}}=(0,0)
$$

in which $\tilde{g}$ is an extension of $g$ on the half-line $\mathbb{R}_{+}$in the class $C_{s+1}^{3+\alpha}\left(\mathbb{R}_{+}, 0\right)$.

Then, the functions $\mathbf{u}=\left(u_{1}, u_{2}\right)=\hat{\zeta} \hat{\mathbf{v}} \equiv(\zeta \mathbf{v}) \circ \Phi, q=\hat{\zeta} \hat{p} \equiv(\zeta p) \circ \Phi, r_{1}=\hat{\zeta} \hat{\rho}_{1} \equiv\left(\zeta \rho_{1}\right) \circ$ $\left.\Phi\right|_{\gamma_{0}}$, and $r_{2}=\left.\hat{\zeta} \hat{\rho}_{2} \equiv\left(\zeta \rho_{2}\right) \circ \Phi\right|_{\gamma_{\theta}}$ (here the same symbols $\mathbf{v}, p, \rho_{1}$, and $\rho_{2}$ are used to denote the unknowns defined in the coordinates $\{y\}$ ), where

$$
\zeta(y) \in C_{0}^{\infty}\left(\mathbf{R}^{2}\right), \quad \zeta=1\left(|y|<\frac{\delta}{2}\right), \quad \zeta=0(|y|>\delta)
$$

for a constant $\delta>0$ satisfy the following equations in the sectorial domain $d_{\theta}$ :

$$
\begin{aligned}
& \left\{\begin{array}{l}
-v \Delta \mathbf{u}+\nabla q=\hat{\zeta} \hat{\mathcal{F}}-v\left(\nabla^{2}-\hat{\nabla}^{2}\right) \mathbf{u}+(\nabla-\hat{\nabla}) q+\mathbf{F}, \\
\nabla \cdot \mathbf{u}=\hat{\zeta} \hat{\mathcal{G}}+(\nabla-\hat{\nabla}) \cdot \mathbf{u}+G \quad \text { in } d_{\theta},
\end{array}\right. \\
& \left\{\begin{array}{l}
2 \nu \mathbf{D}(\mathbf{u}) \mathbf{n} \cdot \tau=\hat{\zeta}\left(\hat{\mathbf{a}}-\gamma \hat{\nabla}_{\Gamma} \hat{\rho}_{1}\right) \cdot \hat{\tau} \\
\quad+2 \nu\{\mathbf{D}(\mathbf{u}) \mathbf{n} \cdot \tau-\hat{\mathbf{D}}(\mathbf{u}) \hat{\mathbf{n}} \cdot \hat{\tau}\}+A, \\
\mathbf{u} \cdot \mathbf{n}=\mathbf{u} \cdot(\mathbf{n}-\hat{\mathbf{n}}), \\
-\kappa_{1} \frac{d^{2} r_{1}}{d \eta_{1}^{2}}+\chi_{1} r_{1}=-\hat{\zeta} \hat{\nabla}_{\Gamma} \cdot \hat{\mathbf{v}} \\
\quad-\kappa_{1}\left(\left(\frac{d}{d \eta_{1}}\right)^{2}-\left(\frac{\hat{d}}{d \eta_{1}}\right)^{2}\right) r_{1}+H_{1} \quad \text { on } \gamma_{0},
\end{array}\right. \\
& \left\{\begin{array}{l}
2 \nu \mathbf{D}(\mathbf{u}) \mathbf{n} \cdot \tau=\hat{\zeta}\left(\hat{\mathbf{b}}-\beta \hat{\mathbf{v}}-\frac{\gamma}{2} \hat{\nabla}_{\Sigma} \hat{\rho}_{2}\right) \cdot \hat{\tau} \\
\quad+2 v\{\mathbf{D}(\mathbf{u}) \mathbf{n} \cdot \tau-\hat{\mathbf{D}}(\mathbf{u}) \hat{\mathbf{n}} \cdot \hat{\tau}\}+B, \\
\mathbf{u} \cdot \mathbf{n}=\mathbf{u} \cdot(\mathbf{n}-\hat{\mathbf{n}}) \\
-\kappa_{2} \cos ^{2} \theta \frac{d^{2} r_{2}}{d \eta_{1}^{2}}+\chi_{2} r_{2}=-\frac{1}{2} \hat{\zeta} \hat{\nabla}_{\Sigma} \cdot \hat{\mathbf{v}} \\
\quad-\kappa_{2} \cos ^{2} \theta\left(\left(\frac{d}{d \eta_{1}}\right)^{2}-\left(\frac{\hat{d}}{d \eta_{1}}\right)^{2}\right) r_{2}+H_{2} \quad \text { on } \gamma_{\theta},
\end{array}\right. \\
& \left\{\begin{array}{c}
-\kappa_{1} \frac{d r_{1}}{d \eta_{1}}=-\hat{\zeta} \hat{\mathbf{v}} \cdot \hat{\mathbf{e}}_{1}-\kappa_{1}\left(\frac{d}{d \eta_{1}}-\frac{\hat{d}}{d \eta_{1}}\right) r_{1}-\kappa_{1} \hat{\rho}_{1}\left(\frac{\hat{d}}{d \eta_{1}}\right) \hat{\zeta}, \\
-\kappa_{2} \cos \theta \frac{d r_{2}}{d \eta_{1}}=-\frac{1}{2} \hat{\zeta} \hat{\mathbf{v}} \cdot \hat{\mathbf{e}}_{2}-\kappa_{2} \cos \theta\left(\frac{d}{d \eta_{1}}-\frac{\hat{d}}{d \eta_{1}}\right) r_{2} \\
-\kappa_{2} \cos \theta \hat{\rho}_{2}\left(\frac{\hat{d}}{d \eta_{1}}\right) \hat{\zeta} \quad \text { on } M \equiv \bar{\gamma}_{0} \cap \bar{\gamma}_{\theta},
\end{array}\right.
\end{aligned}
$$

where

$$
\left\{\begin{array}{l}
\mathbf{F}=-v\left\{2(\hat{\nabla} \hat{\zeta} \cdot \hat{\nabla}) \hat{\mathbf{v}}+\hat{\mathbf{v}} \hat{\nabla}^{2} \hat{\zeta}\right\}+(\hat{\nabla} \hat{\zeta}) \hat{p}, \quad G=\hat{\nabla} \hat{\zeta} \cdot \hat{\mathbf{v}} \\
A=B=v\{\hat{\nabla} \hat{\zeta}(\hat{\mathbf{v}} \cdot \hat{\mathbf{n}})+\hat{\mathbf{v}}(\hat{\nabla} \hat{\zeta} \cdot \hat{\mathbf{n}})-2(\hat{\mathbf{v}} \cdot \hat{\mathbf{n}})(\hat{\nabla} \hat{\zeta} \cdot \hat{\mathbf{n}}) \hat{\mathbf{n}}\}, \\
H_{1}=-\kappa_{1}\left\{\left(\frac{\hat{d}}{d \eta_{1}}\right)^{2} r_{1}-\hat{\zeta} \mathcal{G}_{1} \frac{\hat{d}}{d \eta_{1}}\left(\mathcal{G}_{2} \frac{\hat{d}}{d \eta_{1}} \hat{\rho}_{1}\right)\right\}, \\
H_{2}=-\kappa_{2} \cos ^{2} \theta\left\{\left(\frac{\hat{d}}{d \eta_{1}}\right)^{2} r_{2}-\hat{\zeta}\left(\frac{\hat{d}}{d \eta_{1}}\right)^{2} \hat{\rho}_{2}\right\} .
\end{array}\right.
$$


When $\theta=\pi / 2$, equations $(67)^{3}$ and $(68)^{2}$ are replaced by $-\kappa_{2} \frac{d^{2} r_{2}}{d \eta_{2}^{2}}+\chi_{2} r_{2}=-\frac{1}{2} \hat{\zeta} \hat{\nabla} \bar{\Sigma} \cdot \hat{\mathbf{v}}$ and $-\kappa_{2} \frac{d r_{2}}{d \eta_{2}}=-\frac{1}{2} \hat{\zeta} \hat{\mathbf{v}} \cdot \hat{\mathbf{e}}_{2}$, respectively.

Here, $\hat{\nabla}=\left({ }^{t} \mathcal{A}^{-1}\right) \nabla$, where $\mathcal{A}$ is the Jacobian matrix of the mapping $\Phi$ and ${ }^{t} A$ denotes the transpose of matrix $A ; \hat{\mathbf{D}}(\hat{\mathbf{v}})$ denotes the tensor with elements $\frac{1}{2} \sum_{k=1}^{3}\left(a_{i k} \partial_{\eta_{k}} \hat{v}_{j}+\right.$ $\left.a_{j k} \partial_{\eta_{k}} \hat{v}_{i}\right)(i, j=1,2)$, where $a_{i j}$ is the $(i, j)$ th element of ${ }^{t} \mathcal{A}^{-1} ; \hat{\nabla}_{\Gamma}$ and $\hat{\nabla}_{\Sigma}$ are defined by $\hat{\nabla}-(\hat{\mathbf{n}} \cdot \hat{\nabla}) \hat{\mathbf{n}} ; \frac{\hat{d}}{d \eta_{1}}=\hat{\partial}_{\eta_{1}}+\frac{d \hat{\tilde{g}}}{d \eta_{1}} \hat{\partial}_{\eta_{2}}\left(\left(\begin{array}{l}\hat{\partial}_{\eta_{1}} \\ \hat{\partial}_{\eta_{2}}\end{array}\right)=\hat{\nabla}\right)$ on $\gamma_{0} ; \frac{\hat{d}}{d \eta_{1}}=\hat{\partial}_{\eta_{1}}+\tan \theta \hat{\partial}_{\eta_{2}}$ on $\gamma_{\theta} ; \mathcal{G}_{1}=$ $\frac{1}{\left(1-y_{1} \sin \theta+y_{2} \cos \theta\right) \sqrt{1+\left(\tilde{g}^{\prime}\right)^{2}}} \circ \Phi$; and $\mathcal{G}_{2}=\frac{1-y_{1} \sin \theta+y_{2} \cos \theta}{\sqrt{1+\left(\tilde{g}^{\prime}\right)^{2}}} \circ \Phi$.

By applying estimate (27) to the system consisting of (65), (66) ${ }^{1,2}$, and (67) $)^{1,2}$ and applying estimate (44) to the system consisting of $(66)^{3},(67)^{3}$, and (68), we have

$$
\begin{aligned}
|\mathbf{v}|_{s, \Omega_{i}^{\prime}, M_{i}^{\prime}}^{(2+\alpha)}+|\nabla p|_{s-2, \Omega_{i}^{\prime}, M_{i}^{\prime}}^{(\alpha)}+\left|\rho_{1}\right|_{s+1, \Gamma_{i}^{\prime}, M_{i}^{\prime}}^{(3+\alpha)}+\left|\rho_{2}\right|_{s+1, \Sigma_{i}^{\prime}, M_{i}^{\prime}}^{(3+\alpha)} \\
\leq C\left(|\mathbf{f}|_{s-2, \Omega_{i}, M_{i}}^{(\alpha)}+|\mathbf{a}|_{s-1, \Gamma_{i}, M_{i}}^{(1+\alpha)}+|\mathbf{b}|_{s-1, \Sigma_{i}, M_{i}}^{(1+\alpha)}\right. \\
\left.\quad+\|\mathbf{v}\|_{2, \Omega_{i}}+\left\|\rho_{1}\right\|_{2, \Gamma_{i}}+\left\|\rho_{2}\right\|_{2, \Sigma_{i}}\right) .
\end{aligned}
$$

Let $\left\{U_{i}\right\}_{i \in I}$ be a finite covering of $\bar{\Omega}_{0,4}$ which is chosen from the set $\left\{U_{i}\right\}_{i \in \mathbb{N}}$. If we assume that each element of $\left\{U_{i}\right\}_{i \in I_{1}(\subset I)}$ satisfies $U_{i} \cap M=\phi$, then the norms $|\cdot|_{s, \Omega_{i}, M}^{(l+\alpha)}$ and $|\cdot|_{\Omega_{i}}^{(l+\alpha)}$ are equivalent for $i \in I_{1}$, since any point in $\Omega_{i}\left(i \in I_{1}\right)$ is away from $M$ by a positive distance. Thus, by summing estimates (60)-(62) and (70) for all $i \in I$ and then using (55), we can obtain the estimate

$$
\begin{aligned}
& |\mathbf{v}|_{s, \Omega_{0,4}, M}^{(2+\alpha)}+|\nabla p|_{s-2, \Omega_{0,4}, M}^{(\alpha)}+\left|\rho_{1}\right|_{s+1, \Gamma, M}^{(3+\alpha)}+\left|\rho_{2}\right|_{s+1, \Sigma_{0,4}, M}^{(3+\alpha)} \\
& \quad \leq C\left(|\mathbf{f}|_{s-2, \Omega, M}^{(\alpha, \mu)}+|\mathbf{a}|_{s-1, \Gamma, M}^{(1+\alpha)}+|\mathbf{b}|_{s-1, \Sigma, M}^{(1+\alpha, \mu)}\right) .
\end{aligned}
$$

In a similar manner, we take estimates (60) and (62) and use (56) in the domain $\Omega(\lambda, 1-\delta)$ to obtain the estimate

$$
\begin{aligned}
|\mathbf{v}|_{\Omega(\lambda, 1-\delta)}^{(2+\alpha)}+|\nabla p|_{\Omega(\lambda, 1-\delta)}^{(\alpha)}+\left|\rho_{2}\right|_{\Sigma(\lambda, 1-\delta)}^{(3+\alpha)} & \\
\leq & C\left(|\mathbf{f}|_{\Omega(\lambda, 1)}^{(\alpha)}+|\mathbf{b}|_{\Sigma(\lambda, 1)}^{(1+\alpha)}+\|\mathbf{v}\|_{2, \Omega(\lambda, 1)}+\left\|\rho_{2}\right\|_{2, \Sigma(\lambda, 1)}\right) \\
\leq & C\left\{|\mathbf{f}|_{\Omega(\lambda, 1)}^{(\alpha)}+|\mathbf{b}|_{\Sigma(\lambda, 1)}^{(1+\alpha)}\right. \\
& \left.+e^{\mu \lambda}\left(|\mathbf{f}|_{s-2, \Omega, M}^{(\alpha, \mu)}+|\mathbf{a}|_{s-1, \Gamma, M}^{(1+\alpha)}+|\mathbf{b}|_{s-1, \Sigma, M}^{(1+\alpha, \mu)}\right)\right\},
\end{aligned}
$$

where $0<\delta<1$ is a constant. Multiplying both sides of (72) by $e^{-\mu \lambda}$ and taking the supremum for $\lambda<-4$, and then noting that the norms $\left|e^{-\mu x_{3}} \mathbf{v}\right|_{\Omega_{3+\delta}}^{(2+\alpha)}$ and $\sup _{\lambda<-4} e^{-\mu \lambda}|\mathbf{v}|_{\Omega(\lambda, 1-\delta)}^{(2+\alpha)}$ are equivalent, we have

$$
\begin{aligned}
& |\mathbf{v}|_{\Omega_{3+\delta}}^{(2+\alpha, \mu)}+|\nabla p|_{\Omega_{3+\delta}}^{(\alpha, \mu)}+\left|\rho_{2}\right|_{\Sigma_{3+\delta}}^{(3+\alpha, \mu)} \\
& \quad \leq C\left(|\mathbf{f}|_{s-2, \Omega, M}^{(\alpha, \mu)}+|\mathbf{a}|_{s-1, \Gamma, M}^{(1+\alpha)}+|\mathbf{b}|_{s-1, \Sigma, M}^{(1+\alpha, \mu)}\right)
\end{aligned}
$$

Thus, from (71) and (73), we reach the desired result.

\section{Nonlinear problem}

In this section, we prove Theorem 1. 
Assume that $W=0$ and $\mathbf{v}=\mathbf{v}_{1}=\mathbf{v}_{2}=\mathbf{0}$. Then, from $(1)^{1},(3)$, and (7), it follows that $p=\bar{p}=$ const., $\rho_{1}=\rho_{1, e}$, and $\rho_{2}=\rho_{2, e}$.

Thus, the functions

$$
\left(\mathbf{v}, p, \rho_{1}, \rho_{2}, h\right)=\left(\mathbf{0}, \bar{p}, \rho_{1, e}, \rho_{2, e}, \bar{h}\right)
$$

describe the rest state of problem $(\mathcal{P})$. Here, $\bar{h}$ is a solution of the equation

$$
\left\{\begin{array}{l}
\bar{\sigma}_{1} \nabla^{\prime} \cdot \frac{\nabla^{\prime} \bar{h}}{\sqrt{1+\left|\nabla^{\prime} \bar{h}\right|^{2}}}=-\bar{p} \quad \text { in } B, \\
\frac{\nabla^{\prime} \bar{h}}{\sqrt{1+\left|\nabla^{\prime} \bar{h}\right|^{2}}} \cdot v=\frac{\sigma_{s g}-\bar{\sigma}_{2}}{\bar{\sigma}_{1}}, \quad \bar{h}=0 \quad \text { on } M,
\end{array}\right.
$$

where $\nabla^{\prime}=\left(\partial_{x_{1}}, \partial_{x_{2}}\right)$ and $v$ is the unit outward normal to $\partial \Omega$ with the starting point located on $M$.

It is easy to see that $\bar{h}$ is defined uniquely by a part of the sphere

$$
x_{1}^{2}+x_{2}^{2}+\left(x_{3}+\tan \bar{\theta}\right)^{2}=\left(\frac{1}{\cos \bar{\theta}}\right)^{2}
$$

with $\cos \bar{\theta}=\left(\sigma_{s g}-\bar{\sigma}_{2}\right) / \bar{\sigma}_{1}$. Note that $\bar{p}$ is uniquely determined as $\bar{p}=-2\left(\sigma_{s g}-\bar{\sigma}_{2}\right)$ from the compatibility condition of problem (74).

We seek a solution of the form

$$
\mathbf{u}=\mathbf{v}-\zeta \mathbf{V}, \quad q=p-\bar{p}-\zeta P, \quad r_{1}=\rho_{1}-\rho_{1, e}, \quad r_{2}=\rho_{2}-\rho_{2, e}, \quad \delta h=h-\bar{h},
$$

where $\zeta$ is a smooth cut-off function satisfying

$$
\zeta(x)=1 \quad \text { if } x_{3} \leq-2, \quad \zeta(x)=0 \quad \text { if } x_{3} \geq-1
$$

In terms of the new unknowns in the above formulation, conditions $(13)^{2}$ and (16) can be written as

$$
\begin{aligned}
\bar{\sigma}_{1} \nabla^{\prime} \cdot \mathbf{A}\left(x^{\prime}\right) \nabla^{\prime} \delta h= & \gamma r_{1} \nabla^{\prime} \cdot \frac{\nabla^{\prime} h}{\sqrt{1+\left|\nabla^{\prime} h\right|^{2}}}-q+2 \nu \mathbf{n} \cdot \mathbf{D}(\mathbf{u}) \mathbf{n} \\
& -\bar{\sigma}_{1} \sum_{j=1,2} \partial_{x_{j}}\left[\nabla^{\prime} \delta h \cdot \int_{0}^{1}(1-\tau) \mathbf{B}^{(j)}\left(\nabla^{\prime} \bar{h}+\tau \nabla^{\prime} \delta h\right) d \tau \nabla^{\prime} \delta h\right] \\
\equiv & \mathcal{F}\left(\mathbf{u}, q, r_{1}, \delta h\right) \quad \text { in } B
\end{aligned}
$$

and

$$
\begin{aligned}
\left.\mathbf{A}\left(x^{\prime}\right) \nabla^{\prime} \delta h \cdot v\right|_{M}= & \frac{\sigma_{3}-\rho_{0}+\rho_{2, e}}{\left(\rho_{0}-\rho_{1, e}-r_{1}\right)\left(\rho_{0}-\rho_{1, e}\right)} r_{1}+\frac{1}{\rho_{0}-\rho_{1, e}-r_{1}} r_{2} \\
& -\sum_{j=1,2} v_{j} \nabla^{\prime} \delta h \cdot \int_{0}^{1}(1-\tau) \mathbf{B}^{(j)}\left(\nabla^{\prime} \bar{h}+\tau \nabla^{\prime} \delta h\right) d \tau \nabla^{\prime} \delta h \\
\equiv & \mathcal{G}\left(r_{1}, r_{2}, \delta h\right)
\end{aligned}
$$


where $\mathbf{A}\left(x^{\prime}\right)$ and $\mathbf{B}^{(j)}\left(\xi^{\prime}\right)$ are matrices whose $(k, l)$ th components are $\left.\partial_{\xi l} F_{k}\right|_{\xi^{\prime}=\nabla^{\prime} h}$ and $\partial_{\xi_{k} \xi_{l}}^{2} F_{j}\left(\xi^{\prime}\right)$, respectively, and $F_{j}\left(\xi^{\prime}\right)=\xi_{j} / \sqrt{1+\left|\xi^{\prime}\right|^{2}}\left(\xi^{\prime}=\left(\xi_{1}, \xi_{2}\right)\right)$.

We now prove the following theorem, which will be applied to the problem consisting of (76) and (77).

Theorem 5 Let $0<\alpha<1, s \notin \mathbb{Z}$, and $0<s \leq 3+\alpha$. Assume that $\bar{h} \in C^{3+\alpha}(B), f \in$ $C_{s-1}^{\alpha+1}(B, M)$, and $g \in C(M)$, and that $\bar{h}, f$, and $g$ are axially symmetric with respect to the $x_{3}$-axis. Further, assume that the compatibility condition

$$
\int_{B} f d x^{\prime}=\int_{M} g d l
$$

is satisfied. Then the problem

$$
\left\{\begin{array}{l}
\nabla^{\prime} \cdot \mathbf{A}\left(x^{\prime}\right) \nabla^{\prime} u=f \quad \text { in } B, \\
\mathbf{A}\left(x^{\prime}\right) \nabla^{\prime} u \cdot v=g \quad \text { on } M
\end{array}\right.
$$

has an axisymmetric solution satisfying the estimate

$$
|u|_{s+1, B, M}^{(3+\alpha)} \leq C|f|_{s-1, B, M}^{(1+\alpha)}
$$

for a constant $C>0$ that is independent of the data. This solution is uniquely determined if $u(P)=0$ is satisfied for a point $P \in M$.

Proof When $s>1$, the above result is readily obtained with the aid of a standard theory of second-order elliptic partial differential equations. Therefore, we consider only the case $0<s<1$.

As the higher-order derivatives of the solution are estimated using Schauder's method, we derive only the following estimate:

$$
|u|_{B}^{(s+1)} \leq C\langle f\rangle_{s-1, B, M}^{(1+\alpha)}
$$

In the polar coordinate system, problem (79) is written as

$$
\left\{\begin{array}{l}
\frac{1}{r}\left(\frac{r}{H} u^{\prime}\right)^{\prime}=f \quad \text { on } I \equiv(0,1), \\
u^{\prime}(1)=H(1) g(1), \quad u(1)=0,
\end{array}\right.
$$

where $H=\left\{1+\left(\frac{\partial \bar{h}}{\partial r}\right)^{2}\right\}^{3 / 2}$.

The following function $u$ satisfies the above problem:

$$
u(r)=\int_{1}^{r} \frac{H(s)}{s} d s \int_{0}^{r} \xi f(\xi) d \xi+\int_{r}^{1}\left(\int_{1}^{\xi} \frac{H(s)}{s} d s\right) \xi f(\xi) d \xi .
$$

From this expression, we have the estimate

$$
|u|_{I}^{(0)} \leq C\langle f\rangle_{s-1, I, 1}^{(1+\alpha)} .
$$


The first term on the right-hand side is estimated as follows:

$$
\begin{aligned}
& \left|\int_{1}^{r} \frac{H(s)}{s} d s \int_{0}^{r} \xi f(\xi) d \xi\right| \\
& \quad \leq C(-\log r)\langle f\rangle_{s-1, I, 1}^{(1+\alpha)} \int_{0}^{r} \xi(1-\xi)^{s-1} d \xi \leq C\langle f\rangle_{s-1, I, 1}^{(1+\alpha)} .
\end{aligned}
$$

The second term is estimated in the same way.

Next, we derive the estimate

$$
\left|u^{\prime \prime}\right|_{I}^{(0)} \leq C(1-r)^{s-1}\langle f\rangle_{s-1, I, 1}^{(1+\alpha)}
$$

The relation $u^{\prime \prime}(r)=\left(\frac{H(r)}{r}\right)^{\prime} \int_{0}^{r} \xi f(\xi) d \xi+H(r) f(r)$ can be used to obtain

$$
\left|u^{\prime \prime}(r)\right| \leq C\left(\frac{1}{r^{2}} \int_{0}^{r} \xi f(\xi) d \xi+(1-r)^{s-1}\langle f\rangle_{s-1, I, 1}^{(1+\alpha)}\right)
$$

The first term on the right-hand side is estimated as

$$
\begin{aligned}
& \frac{1}{r^{2}}\left|\int_{0}^{r} \xi f(\xi) d \xi\right| \\
& \quad \leq \frac{C}{r^{2}}(1-r)^{s-1}\langle f\rangle_{s-1, I, 1}^{(1+\alpha)} \int_{0}^{r} \xi d \xi \leq C(1-r)^{s-1}\langle f\rangle_{s-1, I, 1}^{(1+\alpha)}
\end{aligned}
$$

Now, let us estimate $\left[u^{\prime}\right]_{I}^{(s)}$. For this purpose, we use the following two relations:

$$
\begin{aligned}
& u^{\prime}(r+\tau)-u^{\prime}(r) \\
& \quad=\left(\frac{H(r+\tau)}{r+\tau}-\frac{H(r)}{r}\right) \int_{0}^{r+\tau} \xi f(\xi) d \xi+\frac{H(r)}{r} \int_{r}^{r+\tau} \xi f(\xi) d \xi
\end{aligned}
$$

and

$$
u^{\prime}(r+\tau)-u^{\prime}(r)=u^{\prime \prime}(t) \tau \quad \text { for a number } t \text { between } r \text { and } r+\tau \text {. }
$$

When $0<r, r+\tau \leq \frac{1}{2}$, from (85), $\left|u^{\prime \prime}(t)\right| \leq C\langle f\rangle_{s-1, I, 1}^{(1+\alpha)}$ holds. Therefore, from (89), we have the estimate

$$
\left|u^{\prime}(r+\tau)-u^{\prime}(r)\right| \leq C \tau^{s}\langle f\rangle_{s-1, I, 1}^{(1+\alpha)}
$$

When $\frac{1}{2} \leq r, r+\tau<1$, with the aid of (88), we have (90). Finally, when $0<r \leq \frac{1}{2}<r+\tau<1$, noting the inequality

$$
\left|u^{\prime}(r+\tau)-u^{\prime}(r)\right| \leq\left|u^{\prime}(r+\tau)-u^{\prime}\left(\frac{1}{2}\right)\right|+\left|u^{\prime}\left(\frac{1}{2}\right)-u^{\prime}(r)\right|,
$$

we have (90).

Thus, we obtain estimate (81). 
The remaining equations are given by

$$
\left\{\begin{array}{l}
-v \Delta \mathbf{u}+\nabla q=\mathbf{f}(\mathbf{u}), \quad \nabla \cdot \mathbf{u}=g \quad \text { in } \Omega_{\delta h}, \\
2 v \Pi \mathbf{D}(\mathbf{u}) \mathbf{n}+\gamma \nabla_{\delta h} r_{1}=\mathbf{0}, \quad \mathbf{u} \cdot \mathbf{n}=0, \\
-\kappa_{1} \nabla_{\delta h}^{2} r_{1}+\chi_{1} r_{1}+\nabla_{\delta h} \cdot \mathbf{u}=h_{1}\left(\mathbf{u}, r_{1}\right) \quad \text { on } \Gamma_{\delta h}, \\
2 v \Pi \mathbf{D}(\mathbf{u}) \mathbf{n}+\frac{\gamma}{2} \nabla_{\Sigma} r_{2}+\beta \Pi \mathbf{u}=\mathbf{b}, \quad \mathbf{u} \cdot \mathbf{n}=b_{3}, \\
-\kappa_{2} \nabla_{\Sigma}^{2} r_{2}+\chi_{2} r_{2}+\frac{1}{2} \nabla_{\Sigma} \cdot \mathbf{u}=h_{2}\left(\mathbf{u}, r_{2}\right) \quad \text { on } \Sigma, \\
\left(\mathbf{u}-\kappa_{1} \nabla_{\delta h} r_{1}\right) \cdot \mathbf{e}_{1}=m_{1}\left(r_{1}, r_{2}\right), \\
\left(\frac{1}{2} \mathbf{u}-\kappa_{2} \nabla_{\Sigma} r_{2}\right) \cdot \mathbf{e}_{2}=m_{2}\left(r_{1}, r_{2}\right) \quad \text { on } M, \\
\mathbf{u} \longrightarrow \mathbf{0}, \quad r_{2} \longrightarrow 0 \quad\left(x_{3} \longrightarrow-\infty\right),
\end{array}\right.
$$

where $\Omega_{\delta h}=\Omega_{h}$ and $\Gamma_{\delta h}=\Gamma_{h}\left(\Omega_{h}\right.$ and $\Gamma_{h}$ are defined in Sect. 2); $\nabla_{\delta h}$ is the gradient operator on $\Gamma_{\delta h}, \kappa_{1}=\chi \gamma, \chi_{1}=1 /\left(\tau_{1} \rho_{1, e}\right), \chi_{2}=1 /\left(\tau_{2} \rho_{2, e}\right), \kappa_{2}=\alpha \gamma$, and

$$
\left\{\begin{aligned}
& \mathbf{f}(\mathbf{u})=-(\mathbf{u}+\zeta \mathbf{V} \cdot \nabla)(\mathbf{u}+\zeta \mathbf{V})+\nu \Delta(\zeta \mathbf{V})-\nabla(\zeta P), \\
& g=-\nabla \cdot \zeta \mathbf{V} \\
& \mathbf{b}=-2 \nu \Pi \mathbf{D}(\zeta \mathbf{V}) \mathbf{n}-\beta \Pi(\zeta \mathbf{V}-\mathbf{W}) \\
& b_{3}=-\zeta \mathbf{V} \cdot \mathbf{n}, \\
& h_{1}\left(\mathbf{u}, r_{1}\right)= \frac{\kappa_{1}}{\rho_{1, e}} \nabla_{\delta h} \cdot r_{1} \nabla_{\delta h} r_{1}-\frac{1}{\rho_{1, e}} \nabla_{\delta h} \cdot r_{1} \mathbf{u}, \\
& h_{2}\left(\mathbf{u}, r_{2}\right)= \frac{\kappa_{2}}{\rho_{2, e}} \nabla_{\Sigma} \cdot r_{2} \nabla_{\Sigma} r_{2} \\
&-\frac{1}{2 \rho_{2, e}} \nabla_{\Sigma} \cdot r_{2}(\mathbf{u}+\zeta \mathbf{V}+\mathbf{W})-\frac{1}{2} \nabla_{\Sigma} \cdot(\zeta \mathbf{V}+\mathbf{W}), \\
& m_{1}\left(r_{1}, r_{2}\right)= \frac{\kappa_{1}}{\left(2 \rho_{2, e} \alpha-\rho_{1, e} \chi\right) \gamma}\left\{\left(\frac{1}{2} \rho_{2, e}-2 \rho_{2, e} \alpha \beta\right) \mathbf{W} \cdot \mathbf{e}_{2}\right. \\
&\left.-\kappa_{1} r_{1} \nabla_{\delta h} r_{1} \cdot \mathbf{e}_{1}+r_{2}\left(\frac{1}{2} \mathbf{W}-\kappa_{2} \nabla_{\Sigma} r_{2}\right) \cdot \mathbf{e}_{2}\right\} \\
& m_{2}\left(r_{1}, r_{2}\right)=-\frac{2 \kappa_{2}}{\left(2 \rho_{2, e} \alpha-\rho_{1, e} \chi\right) \gamma}\left\{\left(\frac{1}{2} \rho_{2, e}-\rho_{1, e} \chi \beta\right) \mathbf{W} \cdot \mathbf{e}_{2}\right. \\
&\left.-\kappa_{1} r_{1} \nabla_{\delta h} r_{1} \cdot \mathbf{e}_{1}+r_{2}\left(\frac{1}{2} \mathbf{W}-\kappa_{2} \nabla_{\Sigma} r_{2}\right) \cdot \mathbf{e}_{2}\right\} .
\end{aligned}\right.
$$

Let $X_{\epsilon}$ and $Y_{\epsilon, \delta h}$ be function spaces defined by

$$
X_{\epsilon} \equiv\left\{\left.\delta h \in C_{s+1}^{3+\alpha}(B, M)|| \delta h\right|_{s+1, B, M} ^{(3+\alpha)} \leq 2 K \epsilon\right\}
$$

and

$$
Y_{\epsilon, \delta h} \equiv\left\{\begin{array}{c}
\left(\mathbf{u}, \nabla q, r_{1}, r_{2}\right) \in C_{s}^{2+\alpha, \mu}\left(\Omega_{\delta h}, M\right) \times C_{s-2}^{\alpha, \mu}\left(\Omega_{\delta h}, M\right) \\
\times C_{s+1}^{3+\alpha}\left(\Gamma_{\delta h}, M\right) \times\left. C_{s+1}^{3+\alpha, \mu}(\Sigma, M)|| \mathbf{u}\right|_{s, \Omega_{\delta h}, M} ^{(2+\alpha, \mu)}+|\nabla q|_{s-2, \Omega_{\delta h}, M}^{(\alpha, \mu)} \\
+\left|r_{1}\right|_{s+1, \Gamma_{\delta h}, M}^{(3+\alpha)}+\left|r_{2}\right|_{s+1, \Sigma, M}^{(3+\alpha, \mu)} \leq \epsilon
\end{array}\right\}
$$

where $\epsilon$ and $K$ are positive constants.

It is easy to show that the following lemmas hold. Note that an additive constant of $q$ is determined from the compatibility condition of the problem consisting of (76) and (77). Thus, the following estimate holds for $q$ : $|q|_{s-1, \Omega_{0,1}, M}^{(1+\alpha)} \leq C\left(|\mathbf{u}|_{s, \Omega_{\delta h}, M}^{(2+\alpha, \mu)}+\left|r_{1}\right|_{s+1, \Gamma_{\delta h}, M}^{(3+\alpha)}+\right.$ $\left.\left|r_{2}\right|_{s+1, \Sigma, M}^{(3+\alpha, \mu)}+|\delta h|_{s+1, B, M}^{(3+\alpha)}\left(|\delta h|_{s+1, B, M}^{(3+\alpha)}+\left|r_{1}\right|_{s+1, \Gamma_{\delta h}, M}^{(3+\alpha)}\right)\right)$. 
Lemma 9 Assume that $\left(\mathbf{u}, \nabla q, r_{1}, r_{2}\right) \in Y_{\epsilon, \delta h}$. Then, for $\delta h \in X_{\epsilon}$, the following inequality holds:

$$
\left|\mathcal{F}\left(\mathbf{u}, q, r_{1}, \delta h\right)\right|_{s-1, B, M}^{(1+\alpha)} \leq C\left(\epsilon+\left(K+K^{2}\right) \epsilon^{2}\right)
$$

where $C$ is a positive constant that is independent of $\epsilon$ and $K$.

Lemma 10 Assume that $0<W<1$ and $\delta h \in X_{\epsilon}$. Then, for $\left(\mathbf{u}, r_{1}, r_{2}\right),\left(\mathbf{u}^{\prime}, r_{1}^{\prime}, r_{2}^{\prime}\right) \in Y_{\epsilon, \delta h}$, the following inequalities hold:

$$
\begin{aligned}
& |\mathbf{f}(\mathbf{u})|_{s-2, \Omega_{\delta h}, M}^{(\alpha, \mu)},\left|h_{2}\left(\mathbf{u}, r_{2}\right)\right|_{s-1, \Sigma, M}^{(1+\alpha, \mu)}, \\
& \left|m_{1}\left(r_{1}, r_{2}\right)\right|_{M}^{(s)},\left|m_{2}\left(r_{1}, r_{2}\right)\right|_{M}^{(s)} \leq C\left(\epsilon^{2}+W\right), \\
& |g|_{s-1, \Omega_{\delta h}, M}^{(1+\alpha, \mu)},|\mathbf{b}|_{s-1, \Sigma, M}^{(1+\alpha, \mu)},\left|b_{3}\right|_{s, \Sigma, M}^{(2+\alpha, \mu)} \leq C W \\
& \left|h_{1}\left(\mathbf{u}, r_{1}\right)\right|_{s-1, \Gamma_{\delta h}, M}^{(1+\alpha)} \leq C \epsilon^{2}, \\
& \left|\mathbf{f}(\mathbf{u})-\mathbf{f}\left(\mathbf{u}^{\prime}\right)\right|_{s-2, \Omega_{\delta h}, M}^{(\alpha, \mu)} \leq C(\epsilon, W)\left|\mathbf{u}-\mathbf{u}^{\prime}\right|_{s-1, \Omega_{\delta h}, M}^{(1+\alpha, \mu)}, \\
& \quad \leq h_{1}\left(\mathbf{u}, r_{1}\right)-\left.h_{1}\left(\mathbf{u}^{\prime}, r_{1}^{\prime}\right)\right|_{s-1, \Gamma_{\delta h}, M} ^{(1+\alpha)} \\
& \quad \leq C(\epsilon)\left(\left|\mathbf{u}-\mathbf{u}^{\prime}\right|_{s, \Omega_{\delta h}, M}^{(2+\alpha, \mu)}+\left|r_{1}-r_{1}^{\prime}\right|_{s+1, \Gamma_{\delta h}, M}^{(3+\alpha)}\right), \\
& \quad h_{2}\left(\mathbf{u}, r_{2}\right)-\left.h_{2}\left(\mathbf{u}^{\prime}, r_{2}^{\prime}\right)\right|_{s-1, \Sigma, M} ^{(1+\alpha, \mu)} \\
& \quad \leq C(\epsilon, W)\left(\left|\mathbf{u}-\mathbf{u}^{\prime}\right|_{s, \Omega_{\delta h}, M}^{(2+\alpha, \mu)}+\left|r_{2}-r_{2}^{\prime}\right|_{s+1, \Sigma, M}^{(3+\alpha, \mu)}\right), \\
& \quad \leq m_{1}\left(r_{1}, r_{2}\right)-\left.m_{1}\left(r_{1}^{\prime}, r_{2}^{\prime}\right)\right|_{M} ^{(s)},\left|m_{2}\left(r_{1}, r_{2}\right)-m_{2}\left(r_{1}^{\prime}, r_{2}^{\prime}\right)\right|_{M}^{(s)} \\
& \quad \leq C(\epsilon, W)\left(\left|r_{1}-r_{1}^{\prime}\right|_{s+1, \Gamma_{\delta h}, M}^{(3+\alpha)}+\left|r_{2}-r_{2}^{\prime}\right|_{s+1, \Sigma, M}^{(3+\alpha, \mu)}\right),
\end{aligned}
$$

where $C$ is a positive constant that is independent of $\epsilon$ and $W$, and $C(\epsilon)$ and $C(\epsilon, W)$ denote positive constants possessing the properties $C(\epsilon) \rightarrow 0$ as $\epsilon \rightarrow 0$ and $C(\epsilon, W) \rightarrow 0$ as $\epsilon, W \rightarrow$ 0 , respectively.

With the aid of the estimates in Lemma 10 and Theorem 2, we have the following lemma. The proof is almost the same as that of Lemma 7 in [11], and is thus omitted.

Lemma 11 There exist $\epsilon>0$ and $W>0$ such that, for any $\delta h \in X_{\epsilon}$, there exists a unique solution $\left(\mathbf{u}, \nabla q, r_{1}, r_{2}\right) \in Y_{\epsilon, \delta h}$ for problem (91).

As the regularity of the solution $\left(\mathbf{u}, \nabla q, r_{1}, r_{2}\right)$ is determined by the size of the contact angle, it depends on $\delta h$. To treat the solutions to problem (91) for various $\delta h \in X_{\epsilon}$ in a common function space, we now assume that the order $s$ satisfies $s=s^{\prime} \equiv \min \left(\bar{\lambda}_{0}, \pi / \bar{\theta}\right)-\delta>$ 0 , where $\delta>0$ is a constant and $\bar{\lambda}_{0}$ is the constant defined in the same way as $\lambda_{0}$ in (24) with $\theta=\bar{\theta}$.

Now, let us introduce a mapping $\Phi_{\delta h}$ defined by

$$
\Phi_{\delta h}: x^{\prime}=y^{\prime}, \quad x_{3}=y_{3}+\chi(y) \delta h\left(y^{\prime}\right)
$$


where $\delta h \in X_{\epsilon}$ and $\chi(y)$ is a smooth function such that

$$
\chi(y)= \begin{cases}1 & \text { if } \bar{h}\left(y^{\prime}\right)-3 K \epsilon \leq y_{3} \leq \bar{h}\left(y^{\prime}\right)+3 K \epsilon \\ 0 & \text { if } y_{3} \leq \bar{h}\left(y^{\prime}\right)-6 K \epsilon, \quad y_{3} \geq \bar{h}\left(y^{\prime}\right)+6 K \epsilon .\end{cases}
$$

For sufficiently small $\epsilon>0, \Phi_{\delta h}$ is injective and maps $\Omega_{\bar{h}}$ onto $\Omega_{\delta h}$, and the Jacobian matrices $\mathcal{J}_{\delta h}$ and $\mathcal{J}_{\delta h^{\prime}}$ of the mappings $\Phi_{\delta h}$ and $\Phi_{\delta h^{\prime}}$ satisfy the estimate

$$
\left|\mathcal{J}_{\delta h}-\mathcal{J}_{\delta h^{\prime}}\right|_{s^{\prime}, \Omega_{\bar{h}}, M}^{(2+\alpha)} \leq C\left|\delta h-\delta h^{\prime}\right|_{s^{\prime}+1, B, M}^{(3+\alpha)}
$$

for a positive constant $C$ that is independent of $\delta h$ and $\delta h^{\prime}$.

We write problem (91) simply as

$$
L_{\delta h}\left(\mathbf{u}, q, r_{1}, r_{2}\right)=R\left(\mathbf{u}, r_{1}, r_{2}\right)
$$

and let $\left(\mathbf{u}, q, r_{1}, r_{2}\right)$ and $\left(\mathbf{u}^{\prime}, q^{\prime}, r_{1}^{\prime}, r_{2}^{\prime}\right)$ be solutions to the problems $L_{\delta h}\left(\mathbf{u}, q, r_{1}, r_{2}\right)=R\left(\mathbf{u}, r_{1}, r_{2}\right)$ and $L_{\delta h^{\prime}}\left(\mathbf{u}^{\prime}, q^{\prime}, r_{1}^{\prime}, r_{2}^{\prime}\right)=R\left(\mathbf{u}^{\prime}, r_{1}^{\prime}, r_{2}^{\prime}\right)$ for given $\delta h$ and $\delta h^{\prime} \in X_{\epsilon}$.

We rewrite these solutions as equations defined in $\Omega_{\bar{h}}$ with the aid of the mappings $\Phi_{\delta h}$ and $\Phi_{\delta h^{\prime}}$, and then subtract one from the other to obtain the following equations:

$$
\begin{aligned}
& -v \Delta\left(\hat{\mathbf{u}}-\tilde{\mathbf{u}^{\prime}}\right)+\nabla\left(\hat{q}-\tilde{q^{\prime}}\right) \\
& =-v\left(\nabla^{2}-\hat{\nabla}^{2}\right)\left(\hat{\mathbf{u}}-\widetilde{\mathbf{u}}^{\prime}\right)+v\left(\hat{\nabla}^{2}-\tilde{\nabla}^{2}\right) \widetilde{\mathbf{u}^{\prime}} \\
& +(\nabla-\hat{\nabla})\left(\hat{q}-\widetilde{q}^{\prime}\right)-(\hat{\nabla}-\tilde{\nabla}) \widetilde{q^{\prime}}+\hat{\mathbf{f}}(\hat{\mathbf{u}})-\tilde{\mathbf{f}}\left(\widetilde{\mathbf{u}^{\prime}}\right) \\
& \equiv \mathbf{F}_{1}\left(\hat{\mathbf{u}}, \widetilde{\mathbf{u}^{\prime}}, \hat{q}, \widetilde{q}^{\prime}, \delta h, \delta h^{\prime}\right), \\
& \nabla \cdot\left(\hat{\mathbf{u}}-\widetilde{\mathbf{u}^{\prime}}\right)=(\nabla-\hat{\nabla}) \cdot\left(\hat{\mathbf{u}}-\widetilde{\mathbf{u}^{\prime}}\right)-(\hat{\nabla}-\tilde{\nabla}) \cdot \widetilde{\mathbf{u}}^{\prime} \\
& +\hat{g}-\tilde{g} \equiv F_{2}\left(\hat{\mathbf{u}}, \widetilde{\mathbf{u}}^{\prime}, \delta h, \delta h^{\prime}\right) \quad \text { in } \Omega_{\bar{h}}, \\
& 2 v \Pi \mathbf{D}\left(\hat{\mathbf{u}}-\widetilde{\mathbf{u}^{\prime}}\right) \mathbf{n}+\gamma \nabla_{\bar{h}}\left(\hat{r}_{1}-\widetilde{r_{1}^{\prime}}\right) \\
& =2 v\left\{\Pi \mathbf{D}\left(\hat{\mathbf{u}}-\widetilde{\mathbf{u}^{\prime}}\right) \mathbf{n}-\hat{\Pi} \hat{\mathbf{D}}\left(\hat{\mathbf{u}}-\widetilde{\mathbf{u}^{\prime}}\right) \hat{\mathbf{n}}-\left(\hat{\Pi} \hat{\mathbf{D}}\left(\widetilde{\mathbf{u}^{\prime}}\right) \hat{\mathbf{n}}-\tilde{\Pi} \tilde{\mathbf{D}}\left(\widetilde{\mathbf{u}^{\prime}}\right) \tilde{\mathbf{n}}\right)\right\} \\
& +\gamma\left(\nabla_{\bar{h}}-\hat{\nabla}_{\delta h}\right)\left(\hat{r}_{1}-\tilde{r}_{1}^{\prime}\right)-\gamma\left(\hat{\nabla}_{\delta h}-\tilde{\nabla}_{\delta h^{\prime}}\right) \tilde{r}_{1}^{\prime} \\
& \equiv \mathbf{F}_{3}\left(\hat{\mathbf{u}}, \widetilde{\mathbf{u}}^{\prime}, \hat{r}_{1}, \widetilde{r_{1}^{\prime}}, \delta h, \delta h^{\prime}\right), \\
& \left(\hat{\mathbf{u}}-\widetilde{\mathbf{u}^{\prime}}\right) \cdot \mathbf{n}=\left(\hat{\mathbf{u}}-\widetilde{\mathbf{u}^{\prime}}\right) \cdot(\mathbf{n}-\hat{\mathbf{n}})-\widetilde{\mathbf{u}^{\prime}} \cdot(\hat{\mathbf{n}}-\tilde{\mathbf{n}}) \\
& \equiv F_{4}\left(\hat{\mathbf{u}}, \widetilde{\mathbf{u}^{\prime}}, \delta h, \delta h^{\prime}\right), \\
& -\kappa_{1} \nabla_{\bar{h}}^{2}\left(\hat{r}_{1}-\tilde{r_{1}^{\prime}}\right)+\chi_{1}\left(\hat{r}_{1}-\widetilde{r_{1}^{\prime}}\right)+\nabla_{\bar{h}} \cdot\left(\hat{\mathbf{u}}-\tilde{\mathbf{u}^{\prime}}\right) \\
& =-\kappa_{1}\left(\nabla_{\bar{h}}^{2}-\hat{\nabla}_{\delta h}^{2}\right)\left(\hat{r}_{1}-\widetilde{r}_{1}^{\prime}\right)+\kappa_{1}\left(\hat{\nabla}_{\delta h}^{2}-\tilde{\nabla}_{\delta h^{\prime}}^{2}\right) \widetilde{r}_{1}^{\prime} \\
& +\left(\nabla_{\bar{h}}-\hat{\nabla}_{\delta h}\right) \cdot\left(\hat{\mathbf{u}}-\widetilde{\mathbf{u}}^{\prime}\right)-\left(\hat{\nabla}_{\delta h}-\tilde{\nabla}_{\delta h^{\prime}}\right) \cdot \widetilde{\mathbf{u}}^{\prime} \\
& +\hat{h}_{1}\left(\hat{\mathbf{u}}, \hat{r}_{1}\right)-\tilde{h}_{1}\left(\widetilde{\mathbf{u}^{\prime}}, \widetilde{r_{1}^{\prime}}\right) \equiv F_{5}\left(\hat{\mathbf{u}}, \widetilde{\mathbf{u}^{\prime}}, \hat{r}_{1}, \widetilde{r_{1}^{\prime}}, \delta h, \delta h^{\prime}\right) \quad \text { on } \Gamma_{\bar{h}} \text {, } \\
& 2 \nu \Pi \mathbf{D}\left(\hat{\mathbf{u}}-\tilde{\mathbf{u}^{\prime}}\right) \mathbf{n}+\frac{\gamma}{2} \nabla_{\Sigma}\left(\hat{r}_{2}-\tilde{r_{2}^{\prime}}\right)+\beta\left(\hat{\mathbf{u}}-\widetilde{\mathbf{u}}^{\prime}\right)=\mathbf{0}, \\
& \left(\hat{\mathbf{u}}-\tilde{\mathbf{u}^{\prime}}\right) \cdot \mathbf{n}=0,
\end{aligned}
$$




$$
\begin{aligned}
& -\kappa_{2} \nabla_{\Sigma}^{2}\left(\hat{r}_{2}-\tilde{r_{2}^{\prime}}\right)+\chi_{2}\left(\hat{r}_{2}-\tilde{r_{2}^{\prime}}\right)+\frac{1}{2} \nabla_{\Sigma} \cdot\left(\hat{\mathbf{u}}-\widetilde{\mathbf{u}^{\prime}}\right) \\
& =\hat{h}_{2}\left(\hat{\mathbf{u}}, \hat{r}_{2}\right)-\tilde{h}_{2}\left(\widetilde{\mathbf{u}^{\prime}},{\widetilde{r_{2}^{\prime}}}_{2}\right) \equiv F_{6}\left(\hat{\mathbf{u}}, \widetilde{\mathbf{u}}^{\prime}, \hat{r}_{2}, \widetilde{r_{2}^{\prime}}\right) \quad \text { on } \Sigma \text {, } \\
& \left\{\left(\hat{\mathbf{u}}-\widetilde{\mathbf{u}^{\prime}}\right)-\kappa_{1} \nabla_{\bar{h}}\left(\hat{r}_{1}-{\widetilde{r_{1}^{\prime}}}_{1}\right)\right\} \cdot \overline{\mathbf{e}}_{1} \\
& =\left(\hat{\mathbf{u}}-\widetilde{\mathbf{u}}^{\prime}\right) \cdot\left(\overline{\mathbf{e}}_{1}-\hat{\mathbf{e}}_{1}\right)-\widetilde{\mathbf{u}}^{\prime} \cdot\left(\hat{\mathbf{e}}_{1}-\tilde{\mathbf{e}}_{1}\right) \\
& -\kappa_{1}\left\{\nabla_{\bar{h}}\left(\hat{r}_{1}-{\widetilde{r_{1}^{\prime}}}_{1}\right) \cdot \overline{\mathbf{e}}_{1}-\hat{\nabla}_{\delta h}\left(\hat{r}_{1}-\widetilde{r}_{1}^{\prime}\right) \cdot \hat{\mathbf{e}}_{1}-\left(\hat{\nabla}_{\delta h}{\widetilde{r^{\prime}}}_{1} \cdot \hat{\mathbf{e}}_{1}-\tilde{\nabla}_{\delta h^{\prime} \tilde{r}_{1}^{\prime}} \cdot \tilde{\mathbf{e}}_{1}\right)\right\} \\
& +\hat{m}_{1}\left(\hat{r}_{1}, \hat{r}_{2}\right)-\tilde{m}_{1}\left(\widetilde{r_{1}^{\prime}}, \widehat{r_{2}^{\prime}}\right) \equiv F_{7}\left(\hat{\mathbf{u}}, \widetilde{\mathbf{u}}^{\prime}, \hat{r}_{1}, \widetilde{r}_{1}^{\prime}, \hat{r}_{2}, \widetilde{r_{2}^{\prime}}, \delta h, \delta h^{\prime}\right), \\
& \left\{\frac{1}{2}\left(\hat{\mathbf{u}}-\widetilde{\mathbf{u}^{\prime}}\right)-\kappa_{2} \nabla_{\Sigma}\left(\hat{r}_{2}-\widetilde{r_{2}^{\prime}}\right)\right\} \cdot \mathbf{e}_{2} \\
& =\hat{m}_{2}\left(\hat{r}_{1}, \hat{r}_{2}\right)-\tilde{m}_{2}\left(\widetilde{r_{1}^{\prime}}, \widetilde{r_{2}^{\prime}}\right) \equiv F_{8}\left(\hat{r}_{1}, \widetilde{r_{1}^{\prime}}, \hat{r}_{2}, \widetilde{r_{2}^{\prime}}\right) \quad \text { on } M \text {. }
\end{aligned}
$$

In the above equations, $\hat{f}$ and $\tilde{f}$ denote the functions $f \circ \Phi_{\delta h}$ and $f \circ \Phi_{\delta h^{\prime}}$, respectively; $\overline{\mathbf{e}}_{1}$ and $\nabla_{\bar{h}}$ are defined for the surface $y_{3}=\bar{h}\left(y^{\prime}\right)$ in the same way as $\mathbf{e}_{1}$ and $\nabla_{\delta h}$, respectively, are defined; $\hat{\Pi}$ is the operator defined by $\hat{\Pi} \mathbf{f}=\mathbf{f}-(\hat{\mathbf{n}} \cdot \mathbf{f}) \hat{\mathbf{n}} ; \hat{\nabla}$ and $\hat{\nabla}_{\delta h}$ are the operators defined by $\hat{\nabla}=\left({ }^{t} \mathcal{J}_{\delta h}\right)^{-1} \nabla$ and $\hat{\nabla}_{\delta h}=\hat{\nabla}-(\hat{\mathbf{n}} \cdot \hat{\nabla}) \hat{\mathbf{n}} ; \hat{\mathbf{D}}(\hat{\mathbf{u}})$ denotes a tensor with elements $(1 / 2) \sum_{k=1}^{3}\left(A_{j k} \partial_{y_{k}} \hat{u}_{i}+A_{i k} \partial_{y_{k}} \hat{u}_{j}\right)(i, j=1,2,3)$, where $A_{i j}$ is the $(i, j)$ th element of $\left({ }^{t} \mathcal{J}_{\delta h}\right)^{-1}$; and the operators $\tilde{\Pi}, \tilde{\nabla}$, and $\tilde{\nabla}_{\delta h^{\prime}}$ and the tensor $\tilde{\mathbf{D}}\left(\widetilde{\mathbf{u}^{\prime}}\right)$, which arise as a result of the change of variables $x=\Phi_{\delta h^{\prime}}(y)$, are defined in the same way.

For the terms on the right-hand sides of (102)-(111), we obtain the estimates given in the following lemma.

Lemma 12 For $\delta h, \delta h^{\prime} \in X_{\epsilon},\left(\mathbf{u}, \nabla q, r_{1}, r_{2}\right) \in Y_{\epsilon, \delta h}$, and $\left(\mathbf{u}^{\prime}, \nabla q^{\prime}, r_{1}^{\prime}, r_{2}^{\prime}\right) \in Y_{\epsilon, \delta h^{\prime}}$, the following inequalities hold:

$$
\begin{aligned}
& \left|\mathbf{F}_{1}\left(\hat{\mathbf{u}}, \widetilde{\mathbf{u}^{\prime}}, \hat{q}, \widetilde{q^{\prime}}, \delta h, \delta h^{\prime}\right)\right|_{s^{\prime}-2, \Omega_{\bar{h}}, M}^{(\alpha, \mu)} \\
& \leq C(\epsilon, W)\left(\left|\hat{\mathbf{u}}-\widetilde{\mathbf{u}^{\prime}}\right|_{s^{\prime}, \Omega_{\bar{h}}, M}^{(2+\alpha, \mu)}+\left|\nabla \hat{q}-\nabla \widetilde{q^{\prime}}\right|_{s^{\prime}-2, \Omega \Omega_{\bar{h}}, M}^{(\alpha, \mu)}+\left|\delta h-\delta h^{\prime}\right|_{s^{\prime}, B, M}^{(2+\alpha)}\right), \\
& \left|F_{2}\left(\hat{\mathbf{u}}, \widetilde{\mathbf{u}^{\prime}}, \delta h, \delta h^{\prime}\right)\right|_{s^{\prime}-1, \Omega_{\bar{h}^{\prime}}, M}^{(1+\alpha, \mu)} \\
& \leq C(\epsilon, W)\left(\left|\hat{\mathbf{u}}-\widetilde{\mathbf{u}^{\prime}}\right|_{s^{\prime}, \Omega_{\bar{h}}, M}^{(2+\alpha, \mu)}+\left|\delta h-\delta h^{\prime}\right|_{\delta^{\prime}, B, M}^{(2+\alpha)}\right), \\
& \left|\mathbf{F}_{3}\left(\hat{\mathbf{u}}, \widetilde{\mathbf{u}^{\prime}}, \hat{r}_{1}, \widetilde{r_{1}^{\prime}}, \delta h, \delta h^{\prime}\right)\right|_{s^{\prime}-1, \Gamma_{h^{\prime}}, M}^{(1+\alpha)} \\
& \leq C(\epsilon)\left(\left|\hat{\mathbf{u}}-\widetilde{\mathbf{u}^{\prime}}\right|_{s^{\prime}, \Omega_{\bar{h}}, M}^{(2+\alpha, \mu)}+\left|\hat{r}_{1}-\widetilde{r_{1}^{\prime}}\right|_{s^{\prime}, \Gamma_{\bar{h}}, M}^{(2+\alpha)}+\left|\delta h-\delta h^{\prime}\right|_{s^{\prime}, B, M}^{(2+\alpha)}\right), \\
& \left|F_{4}\left(\hat{\mathbf{u}}, \widetilde{\mathbf{u}^{\prime}}, \delta h, \delta h^{\prime}\right)\right|_{s^{\prime}, \Gamma_{\bar{h}}, M}^{(2+\alpha)} \\
& \leq C(\epsilon)\left(\left|\hat{\mathbf{u}}-\widetilde{\mathbf{u}^{\prime}}\right|_{s^{\prime}, \Omega_{\bar{h}}, M}^{(2+\alpha, \mu)}+\left|\delta h-\delta h^{\prime}\right|_{s^{\prime}+1, B, M}^{(3+\alpha)}\right), \\
& \left|F_{5}\left(\hat{\mathbf{u}}, \widetilde{\mathbf{u}^{\prime}}, \hat{r}_{1}, \widetilde{r_{1}^{\prime}}, \delta h, \delta h^{\prime}\right)\right|_{s^{\prime}-1, \Gamma_{\bar{h}}, M}^{(1+\alpha)} \\
& \leq C(\epsilon)\left(\left|\hat{\mathbf{u}}-\widetilde{\mathbf{u}^{\prime}}\right|_{s^{\prime}, \Omega_{\bar{h}}, M}^{(2+\alpha, \mu)}+\left|\hat{r}_{1}-\widetilde{r_{1}^{\prime}}\right|_{s^{\prime}+1, \Gamma_{\bar{h}}, M}^{(3+\alpha)}+\left|\delta h-\delta h^{\prime}\right|_{s^{\prime}+1, B, M}^{(3+\alpha)}\right), \\
& \left|F_{6}\left(\hat{\mathbf{u}}, \tilde{\mathbf{u}}^{\prime}, \hat{r}_{2},{\widetilde{r_{2}^{\prime}}}_{2}\right)\right|_{s^{\prime}-1, \Sigma, M}^{(1+\alpha, \mu)} \\
& \leq C(\epsilon, W)\left(\left|\hat{\mathbf{u}}-\widetilde{\mathbf{u}^{\prime}}\right|_{s^{\prime}, \Omega_{\bar{h}^{\prime}}, M}^{(2+\alpha, \mu)}+\mid \hat{r}_{2}-{\widetilde{r_{2}^{\prime}}}_{\left.\right|_{s^{\prime}+1, \Sigma, M}}^{(3+\alpha, \mu)}\right),
\end{aligned}
$$




$$
\begin{aligned}
& \left|F_{7}\left(\hat{\mathbf{u}}, \widetilde{\mathbf{u}}^{\prime}, \hat{r}_{1}, \widetilde{r_{1}^{\prime}}, \hat{r}_{2}, \widetilde{r_{2}^{\prime}}, \delta h, \delta h^{\prime}\right)\right|_{M}^{\left(s^{\prime}\right)} \\
& \leq C(\epsilon, W)\left(\left|\hat{\mathbf{u}}-\widetilde{\mathbf{u}^{\prime}}\right|_{s^{\prime}, \Omega_{\bar{h}}, M}^{(2+\alpha, \mu)}+\left|\hat{r}_{1}-\widetilde{r_{1}^{\prime}}\right|_{s^{\prime}+1, \Gamma_{\bar{h}}, M}^{(3+\alpha)}\right. \\
& \left.+\left|\hat{r}_{2}-\widetilde{r_{2}^{\prime}}\right|_{s^{\prime}+1, \Sigma, M}^{(3+\alpha, \mu)}+\left|\delta h-\delta h^{\prime}\right|_{s^{\prime}+1, B, M}^{(3+\alpha)}\right), \\
& \left|F_{8}\left(\hat{r}_{1}, \widetilde{r_{2}^{\prime}}, \hat{r}_{2}, \widetilde{r_{2}^{\prime}}\right)\right|_{M}^{\left(s^{\prime}\right)} \\
& \leq C(\epsilon, W)\left(\left|\hat{r}_{1}-\widetilde{r_{1}^{\prime}}\right|_{s^{\prime}+1, \Gamma_{\bar{h}}, M}^{(3+\alpha)}+\left|\hat{r}_{2}-\widetilde{r_{2}^{\prime}}\right|_{s^{\prime}+1, \Sigma, M}^{(3+\alpha, \mu)}\right),
\end{aligned}
$$

where $C(\epsilon)$ and $C(\epsilon, W)$ denote positive constants possessing the same property as the corresponding expressions in Lemma 10.

With the aid of the above estimates, applying Theorem 2 to the problem given in (102)(111) yields the following estimate for sufficiently small $\epsilon$ and $W$ :

$$
\begin{aligned}
& \left|\hat{\mathbf{u}}-{\widetilde{\mathbf{u}^{\prime}}}_{S_{s^{\prime}, \Omega_{\bar{h}}, M}}^{(2+\alpha, \mu)}+\right| \nabla \hat{q}-\left.\nabla \widetilde{q^{\prime}}\right|_{s^{\prime}-2, \Omega_{\bar{h}}, M} ^{(\alpha, \mu)}+\left|\hat{r}_{1}-\widetilde{r_{1}^{\prime}}\right|_{s^{\prime}+1, \Gamma_{\bar{h}}, M}^{(3+\alpha)} \\
& +\left|\hat{r}_{2}-\widetilde{r_{2}^{\prime}}\right|_{s^{\prime}+1, \Sigma, M}^{(3+\alpha, \mu)} \leq C(\epsilon, W)\left|\delta h-\delta h^{\prime}\right|_{s^{\prime}+1, B, M}^{(3+\alpha)} .
\end{aligned}
$$

We now turn to the problem given by (76)-(77). For notational simplicity, we represent the problem as $\mathcal{L}(\delta h)=\mathcal{R}\left(\mathbf{u}, q, r_{1}, r_{2}, \delta h\right)$.

Let the mapping $F$ map each $\delta h \in X_{\epsilon}$ to the solution $\tilde{\delta h}$ of the problem

$$
\mathcal{L}(\tilde{\delta h})=\mathcal{R}\left(\mathbf{u}, q, r_{1}, r_{2}, \delta h\right)
$$

where $\left(\mathbf{u}, q, r_{1}, r_{2}\right) \in Y_{\epsilon, \delta h}$ is a solution to problem (91) defined for $\delta h$ given above.

Then, with the aid of Theorem 5 and estimate (93), we have

$$
|\tilde{\delta h}|_{s^{\prime}+1, B, M}^{(3+\alpha)} \leq C_{1}\left(\epsilon+\left(K+K^{2}\right) \epsilon^{2}\right)
$$

Thus, if we choose $C_{1}$ as $K$ and choose $\epsilon$ such that $\left(K+K^{2}\right) \epsilon<1 / 2$, we obtain $|\tilde{\delta h}|_{S^{\prime}+1, B, M}^{(3+\alpha)} \leq$ $(3 / 2) K \epsilon$. This implies that $F$ maps $X_{\epsilon}$ into itself.

Next, we consider the problem

$$
\mathcal{L}\left(\tilde{\delta h}-\widetilde{\delta h^{\prime}}\right)=\mathcal{R}\left(\mathbf{u}, q, r_{1}, r_{2}, \delta h\right)-\mathcal{R}\left(\mathbf{u}^{\prime}, q^{\prime}, r_{1}^{\prime}, r_{2}^{\prime}, \delta h^{\prime}\right)
$$

where $\left(\mathbf{u}^{\prime}, q^{\prime}, r_{1}^{\prime}, r_{2}^{\prime}\right) \in Y_{\epsilon, \delta h^{\prime}}$ is the solution to problem (91) with $\delta h=\delta h^{\prime}$.

Then, with the aid of estimate (120), we have

$$
\begin{aligned}
\left|\tilde{\delta h}-\widetilde{\delta h^{\prime}}\right|_{s^{\prime}+1, B, M}^{(3+\alpha)} & \leq\left|\mathcal{F}\left(\mathbf{u}, q, r_{1}, \delta h\right)-\mathcal{F}\left(\mathbf{u}^{\prime}, q^{\prime}, r_{1}^{\prime}, \delta h^{\prime}\right)\right|_{s^{\prime}-1, B, M}^{(1+\alpha)} \\
& \leq C(\epsilon, W)\left|\delta h-\delta h^{\prime}\right|_{s^{\prime}+1, B, M}^{(3+\alpha)}
\end{aligned}
$$

Thus, if we choose $\epsilon$ and $W$ such that $C(\epsilon, W)<1$, the estimate indicates that $F$ is a contraction in $X_{\epsilon}$. Thus, by the contraction mapping principle, we have proved the existence of a solution to our problem. 
Finally, we prove that the solution constructed above belongs to the classes stated in Theorem 1. For that purpose, it is convenient to rewrite problem (91) in cylindrical coordinates:

$$
\left\{\begin{array}{l}
-v\left(\partial_{r}^{2}+\partial_{z}^{2}\right) u_{r}+\partial_{r} q=v\left(\frac{1}{r} \partial_{r} u_{r}-\frac{u_{r}}{r^{2}}\right)+f_{r}(\mathbf{u}) \equiv F_{r}, \\
-v\left(\partial_{r}^{2}+\partial_{z}^{2}\right) u_{z}+\partial_{z} q=\frac{v}{r} \partial_{r} u_{z}+f_{z}(\mathbf{u}) \equiv F_{z}, \\
\partial_{r} u_{r}+\partial_{z} u_{z}=-\frac{u_{r}}{r}+g \quad \text { in } \Omega, \\
2 v \mathbf{D}(\mathbf{u}) \mathbf{n} \cdot \tau=-\gamma \nabla_{\delta h} r_{1} \cdot \tau, \quad \mathbf{u} \cdot \mathbf{n}=0 \\
-\frac{\kappa_{1}}{r \sqrt{1+\left(h^{\prime}\right)^{2}}} \frac{d}{d r}\left(\frac{r}{\sqrt{1+\left(h^{\prime}\right)^{2}}} \frac{d r_{1}}{d r}\right)+\chi_{1} r_{1} \\
\quad=-\nabla_{\delta h} \cdot \mathbf{u}+h_{1}\left(\mathbf{u}, r_{1}\right) \quad \text { on } \Gamma, \\
2 v \mathbf{D}(\mathbf{u}) \mathbf{n} \cdot \tau=-\left(\beta \mathbf{u}+\frac{\gamma}{2} \nabla_{\Sigma} r_{2}-\mathbf{b}\right) \cdot \tau, \quad \mathbf{u} \cdot \mathbf{n}=b_{3}, \\
-\kappa_{2} \frac{d^{2} r_{2}}{d z^{2}}+\chi_{2} r_{2}=-\frac{1}{2} \nabla_{\Sigma} \cdot \mathbf{u}+h_{2}\left(\mathbf{u}, r_{2}\right) \quad \text { on } \Sigma, \\
\frac{\kappa_{1}}{\sqrt{1+h^{\prime}(1)^{2}}} \frac{d r_{1}}{d r}=-\mathbf{u} \cdot \mathbf{e}_{1}+m_{1}\left(r_{1}, r_{2}\right), \\
\kappa_{2} \frac{d r_{2}}{d z}=-\frac{1}{2} \mathbf{u} \cdot \mathbf{e}_{2}+m_{2}\left(r_{1}, r_{2}\right) \quad \text { at } M .
\end{array}\right.
$$

Here, $\Omega, \Gamma, \mathbf{n}, \tau$, and $\mathbf{e}_{1}$ are defined in the same way as in (64) for $h=\bar{h}+\delta h$.

After localizing the above problem near the contact point $M$ in the same manner as used to obtain (65)-(68), we have the following equations for the functions $\mathbf{w}=\left(w_{1}, w_{2}\right)=\hat{\zeta} \hat{\mathbf{u}}$, $\pi=\hat{\zeta} \hat{q}, s_{1}=\hat{\zeta} \hat{r}_{1}$, and $s_{2}=\hat{\zeta} \hat{r}_{2}$ :

$$
\left\{\begin{array}{l}
-v \Delta w_{1}+\partial_{\eta_{1}} \pi=H_{1}(\hat{\mathbf{u}}, \hat{q})+L_{1}(\hat{\mathbf{u}}, \hat{q}), \\
-v \Delta w_{2}+\partial_{\eta_{2}} \pi=H_{2}(\hat{\mathbf{u}}, \hat{q})+L_{2}(\hat{\mathbf{u}}, \hat{q}), \\
\nabla \cdot \mathbf{w}=H_{3}(\hat{\mathbf{u}})+L_{3}(\hat{\mathbf{u}}) \quad \text { in } d_{\theta_{d}}, \\
2 v \mathbf{D}(\mathbf{w}) \mathbf{n} \cdot \tau=H_{4}(\hat{\mathbf{u}})+L_{4}\left(\hat{\mathbf{u}}, \hat{r}_{1}\right), \\
\mathbf{w} \cdot \mathbf{n}=H_{5}(\hat{\mathbf{u}})+L_{5}(\hat{\mathbf{u}}), \\
-\kappa_{1} \frac{d^{2} s_{1}}{d \eta_{1}^{2}}+\chi_{1} s_{1}=H_{6}\left(\hat{r}_{1}\right)+L_{6}\left(\hat{\mathbf{u}}, \hat{r}_{1}\right) \quad \text { on } \gamma_{0}, \\
2 v \mathbf{D}(\mathbf{w}) \mathbf{n} \cdot \tau=H_{7}(\hat{\mathbf{u}})+L_{7}\left(\hat{\mathbf{u}}, \hat{r}_{2}\right), \\
\mathbf{w} \cdot \mathbf{n}=H_{8}(\hat{\mathbf{u}})+L_{8}(\hat{\mathbf{u}}), \\
-\kappa_{2} \cos ^{2} \theta_{d} \frac{d^{2} s_{2}}{d \eta_{1}^{2}}+\chi_{2} s_{2}=H_{9}\left(\hat{r}_{2}\right)+L_{9}\left(\hat{\mathbf{u}}, \hat{r}_{2}\right) \quad \text { on } \gamma_{\theta_{d}}, \\
-\kappa_{1} \frac{d s_{1}}{d \eta_{1}}=H_{10}\left(\hat{r}_{1}\right)+L_{10}\left(\hat{\mathbf{u}}, \hat{r}_{1}, \hat{r}_{2}\right), \\
-\kappa_{2} \cos \theta_{d} \frac{d s_{2}}{d \eta_{1}}=H_{11}\left(\hat{r}_{2}\right)+L_{11}\left(\hat{\mathbf{u}}, \hat{r}_{1}, \hat{r}_{2}\right) \quad \text { at } M \equiv \overline{\gamma_{0}} \cap \overline{\gamma_{d}} .
\end{array}\right.
$$

Here, $H_{i}(i=1, \ldots, 11)$ are the terms involving the highest-order derivatives of $\mathbf{u}, q, r_{1}$, and $r_{2}$, while $L_{i}(i=1, \ldots, 11)$ comprises the remaining terms. For example, $H_{1}$ and $L_{1}$ are specifically expressed as follows:

$$
\left\{\begin{aligned}
H_{1}(\hat{\mathbf{u}}, \hat{q})= & -v \hat{\zeta} \sum_{i, j, k=1,2}\left(\delta_{i j} \delta_{i k}-a_{i j} a_{i k}\right) \partial_{\eta_{j}} \partial_{\eta_{k}} \hat{u}_{1} \\
& +\hat{\zeta} \sum_{i=1,2}\left(\delta_{1 i}-a_{1 i}\right) \partial_{\eta_{i}} \hat{q}, \\
L_{1}(\hat{\mathbf{u}}, \hat{q})= & -v \sum_{i, j, k=1,2}\left\{( \delta _ { i j } \delta _ { i k } - a _ { i j } a _ { i k } ) \left(\hat{u}_{1} \partial_{\eta_{j}} \partial_{\eta_{k}} \hat{\zeta}+\partial_{\eta_{j}} \hat{\zeta} \partial_{\eta_{k}} \hat{u}_{1}\right.\right. \\
& \left.\left.+\partial_{\eta_{j} j} \hat{u}_{1} \partial_{\eta_{k}} \hat{\zeta}\right)-a_{i j}\left(\partial_{\eta_{j}} a_{i k}\right)\left(\hat{u}_{1} \partial_{\eta_{k}} \hat{\zeta}+\hat{\zeta} \partial_{\eta_{k}} \hat{u}_{1}\right)\right\}+\hat{q} \sum_{i=1,2}\left(\delta_{1 i}-a_{1 i}\right) \partial_{\eta_{i}} \hat{\zeta} \\
& -2 v \hat{\nabla} \hat{\zeta} \cdot \hat{\nabla} \hat{u}_{1}-v \hat{u}_{1} \hat{\nabla}^{2} \zeta+\hat{q} \hat{\partial}_{\eta_{1}} \hat{\zeta}+\hat{\zeta} \hat{F}_{1},
\end{aligned}\right.
$$


where $u_{1}=-\left(u_{r}-1\right) \sin \theta-u_{z} \cos \theta, F_{1}=-\left(F_{r}-1\right) \sin \theta-F_{z} \cos \theta, a_{i j}$ denotes the $(i, j)$ th component of $\left({ }^{t} \mathcal{A}\right)^{-1}$, in which $\mathcal{A}$ is the Jacobian matrix of the mapping $\Phi$, and $\delta_{i j}$ denotes the Kronecker delta.

We note that $\delta_{i j} \delta_{i k}-a_{i j} a_{i k}$ (the coefficients of the highest-order derivatives of $\mathbf{u}$ ) vanish at the origin and that $\delta_{i j} \delta_{i k}-a_{i j} a_{i k} \in C_{s^{\prime}}^{2+\alpha}\left(d_{\theta_{d}}, M\right)$, from which we obtain

$$
\left|\delta_{i j} \delta_{i k}-a_{i j} a_{i k}\right| \leq C|\eta|^{s^{\prime}}
$$

With the aid of this inequality, it is easily found that $\left(\delta_{i j} \delta_{i k}-a_{i j} a_{i k}\right) \partial_{\eta_{i}, \eta_{j}}^{2} \hat{u}_{1} \in \stackrel{\circ}{C}_{2 s^{\prime}-2}^{\alpha}\left(d_{\theta_{d}}, M\right)$; therefore, $H_{1}+L_{1} \in C_{2 s^{\prime}-2}^{\alpha}\left(d_{\theta_{d}}, M\right)$. In a similar manner (we also use the fact that $\hat{\mathbf{u}} \in$ $\stackrel{\circ}{C}_{s^{\prime}}^{2+\alpha}\left(d_{\theta_{d}}, M\right)$ to estimate $\left.H_{5}(\hat{\mathbf{u}})=\hat{\zeta} \hat{\mathbf{u}} \cdot(\mathbf{n}-\hat{\mathbf{n}})\right)$, we see that the terms $H_{i}+L_{i}(i=2, \ldots, 11)$ are smoother by the order of $s^{\prime}$ than the highest-order derivatives of the solutions involved in each $H_{i}$. Therefore, the solution $\left(\mathbf{w}, \nabla \pi, s_{1}, s_{2}\right)$ becomes smoother by the same order:

$$
\left(\mathbf{w}, \nabla \pi, s_{1}, s_{2}\right) \in C_{2 s^{\prime}}^{2+\alpha}\left(d_{\theta_{d}}, M\right) \times C_{2 s^{\prime}-2}^{\alpha}\left(d_{\theta_{d}}, M\right) \times C_{2 s^{\prime}+1}^{3+\alpha}\left(\gamma_{\theta_{0}}, M\right) \times C_{2 s^{\prime}+1}^{3+\alpha}\left(\gamma_{\theta_{d}}, M\right) .
$$

As a result, we have

$$
\begin{aligned}
\left(\mathbf{u}, \nabla q, q, r_{1}, r_{2}\right) \in & C_{2 s^{\prime}}^{2+\alpha, \mu}\left(\Omega_{h}, M\right) \times C_{2 s^{\prime}-2}^{\alpha, \mu}\left(\Omega_{h}, M\right) \times C_{2 s^{\prime}-1}^{1+\alpha}\left(\Omega_{0,1}, M\right) \\
& \times C_{2 s^{\prime}+1}^{3+\alpha}\left(\Gamma_{h}, M\right) \times C_{2 s^{\prime}+1}^{3+\alpha, \mu}(\Sigma, M) .
\end{aligned}
$$

Then, from equations (76)-(77), we have $\delta \in C_{2 s^{\prime}+1}^{3+\alpha}(B, M)$. By repeating this procedure, we reach the desired result.

\section{Conclusion}

A free boundary problem describing a steadily advancing meniscus in an infinite circular tube has been investigated. The problem was formulated using the interface formation model, and it was proved that an axisymmetric solution exists in weighted Hölder spaces when the velocity of the meniscus is low. The problem was closed by adding a condition stating that the pressure must be finite at the contact line. Our analysis shows that this treatment determines the contact angle as part of the solution and removes the singularity caused by the motion of the contact line (with the exception of the case in which the contact angle is $\pi / 2$ ). It was also shown that the singularity of the solution is caused by the presence of a corner of the domain, and the regularity of the solution near the contact line is determined by the size of the contact angle. For a technical reason, this paper neglected the term representing the mass exchange between the bulk and the interface arising in the normal component of the velocity on the interface. Shikhmurzaev [24] has suggested that the corner of the domain causes no singularity if this term is not neglected-a rigorous verification of this point will be considered in future work.

Acknowledgements

We thank the anonymous reviewers for their valuable comments to improve the quality of the manuscript. We also thank Stuart Jenkinson, PhD, from Edanz (https://jp.edanz.com/ac) for editing a draft of the manuscript.

Funding

This research received no external funding. 


\section{Declarations}

Competing interests

The author declares that he has no competing interests.

\section{Author's contributions}

The author proposed the main idea of this paper and performed all the steps of the proofs in this research. He read and approved the final manuscript.

\section{Publisher's Note}

Springer Nature remains neutral with regard to jurisdictional claims in published maps and institutional affiliations.

Received: 4 September 2021 Accepted: 8 December 2021 Published online: 03 January 2022

\section{References}

1. Billingham, J.: On a model for the motion of a contact line on a smooth solid surface. Eur. J. Appl. Math. 17(3), 347-382 (2006)

2. Blake, T.D., Bracke, M., Shikhmurzaev, Y.D.: Experimental evidence of nonlocal hydrodynamic influence on the dynamic contact angle. Phys. Fluids 11, 1995-2007 (1999)

3. Decent, S.P.: Hydrodynamic assist and the dynamic contact angle in the coalescence of liquid drops. IMA J. Appl. Math. 71(5), 740-767 (2006)

4. Decent, S.P.: The spreading of a viscous microdrop on a solid surface. Microfluid. Nanofluid. 2, 537-549 (2006)

5. DussanV, E.B., Davis, S.H.: On the motion of a fluid-fluid interface along a solid surface. J. Fluid Mech. 65, 71-95 (1974)

6. Galdi, G.P.: An Introduction to the Mathematical Theory of the Navier-Stokes Equations. Vol. I. Linearized Steady Problems. Springer, New York (1994)

7. Griebel, M., Klitz, M.: Simulation of droplet impact with dynamic contact angle boundary conditions. In: Griebel, M. (ed.) Singular Phenomena and Scaling in Mathematical Models, pp. 297-325. Springer, Berlin (2014)

8. Griebel, M., Klitz, M.: Simulation of micron-droplet impact. Comput. Math. Appl. 78, 3027-3043 (2019)

9. Guo, Y., Tice, I.: Stability of contact lines in fluids: 2D Stokes flow. Arch. Ration. Mech. Anal. 227(2), 767-854 (2018)

10. Kozlov, V.A., Mazya, V.G., Rossmann, J.: Elliptic Boundary Value Problems in Domains with Point Singularities. Math Surv. Monogr., vol. 52. Am. Math. Soc., Providence (1997)

11. Kusaka, Y.: Classical solvability of a stationary free boundary problem for an incompressible viscous fluid describing the process of interface formation. Anal. Math. Phys. 5(1), 67-86 (2015)

12. Kusaka, Y.: Classical solvability of the stationary free boundary problem describing the interface formation between two immiscible fluids. Anal. Math. Phys. 6(2), 109-140 (2016)

13. Ladyzhenskaya, O.A., Solonnikov, V.A.: Some problems of vector analysis and generalized formulations of boundary-value problems for the Navier-Stokes equations. Zap. Nauč. Semin. POMI 59, 81-116 (1976); English transl. in J. Soviet Math. 10, 257-286 (1978)

14. Lax, P.D., Milgram, A.N.: Parabolic equations. In: Contributions to the Theory of Partial Differential Equations. Annals of Mathematics Studies, vol. 33, pp. 167-190. Princeton University Press, Princeton (1954)

15. Maz'ya, V.G., Plamenevskiï, B.A.: Estimates in Lp and in Hölder classes, and the Miranda-Agmon maximum principle for the solutions of elliptic boundary-value problems in domains with singular points' on the boundary. Math. Nachr. 81, 25-82 (1978) (Russian); English transl. in Amer. Math. Soc. Transl. 123, 1-56 (1984)

16. Monnier, J., Benselama, A.M., Cotoi, I.: Flow patterns in the vicinity of triple line dynamics arising from a local surface tension model. Int. J. Multiscale Comput. Eng. 5, 417-434 (2007)

17. Monnier, J., Witomski, P.: Analysis of a local hydrodynamic model with Marangoni effect. J. Sci. Comput. 21(3), 369-403 (2004)

18. Mucha, P.B.: On Navier-Stokes equations with slip boundary conditions in an infinite pipe. Acta Appl. Math. 76, 1-15 (2003)

19. Pileckas, K.: Solvability of a problem on a plane motion of a viscous incompressible liquid with a noncompact free boundary. Differ. Uravn. Primen. 30, 57-96 (1981)

20. Sattinger, D.: On the free surface of a viscous fluid motion. Proc. R. Soc. Lond. A 349, 183-204 (1976)

21. Schweizer, B.: A well-posed model for dynamic contact angles. Nonlinear Anal. 43(1), 109-125 (2001)

22. Shikhmurzaev, Y.D.: The moving contact line on a smooth solid surface. Int. J. Multiph. Flow 19(4), 589-610 (1993)

23. Shikhmurzaev, Y.D.: Mathematical modeling of wetting hydrodynamics. Fluid Dyn. Res. 13, 45-64 (1994)

24. Shikhmurzaev, Y.D.: Singularities at the moving contact line. Mathematical, physical and computational aspects. Phys. D, Nonlinear Phenom. 217, 121-122 (2006)

25. Shikhmurzaev, Y.D.: Capillary Flows with Forming Interfaces. Chapman and Hall/CRC, Boca Raton (2008)

26. Sibley, D.N., Savva, N., Kalliadasis, S.: Slip or not slip? A methodical examination of the interface formation model using two-dimensional droplet spreading on a horizontal planar substrate as a prototype system. Phys. Fluids 24 082105 (2012)

27. Socolowsky, J.: Solvability of a stationary problem on the plane motion of two viscous incompressible liquids with noncompact free boundaries. Z. Angew. Math. Mech. 72, 251-268 (1992)

28. Socolowsky, J.: The solvability of a free boundary problem for the stationary Navier-Stokes equations with a dynamic contact line. Nonlinear Anal. 21(10), 763-784 (1993)

29. Solonnikov, V.A.: On the Stokes equations in domains with non-smooth boundaries and on viscous incompressible flow with a free surface. In: Nonlinear Partial Differential Equations and Their Applications. College de France Seminar, Vol. III (Paris, 1980/1981), Res. Notes in Math., vol. 70, pp. 340-423. Pitman, Boston (1982)

30. Solonnikov, V.A.: Solvability of a three-dimensional boundary value problem with a free surface for the stationary Navier-Stokes system. In: Partial Differential Equations, vol. 10, pp. 361-403. Banach Center Publ. (1983) 
31. Solonnikov, V.A.: On the solvability of some two-dimensional quasi-stationary problems with a free boundary for Navierâ-Stokes equations with moving contact point. Zap. Nauč. Semin. POMI 206, 119-206 (1993); Englishi transl. in J. Math. Sci. 80(4), 1951-1955 (1996)

32. Solonnikov, V.A.: On some free boundary problems for the Navierâ-Stokes equations with moving contact points and lines. Math. Ann. 302(4), 743-772 (1995)

33. Solonnikov, V.A., Ščadilov, V.E.: A certain boundary value problem for the stationary system of Navier-Stokes equations. Tr. Mat. Inst. Steklova 125, 196-210 (1973); English transl. in Proc. Steklov Inst. Math. 125, 186-199 (1973)

34. Sprittles, J.E., Shikhmurzaev, Y.D.: Finite element simulation of dynamic wetting flows as an interface formation process. J. Comput. Phys. 233, 34-65 (2013)

35. Zheng, Y., Tice, I.: Local well posedness of the near-equilibrium contact line problem in 2-dimensional Stokes flow. SIAM J. Math. Anal. 49(2), 899-953 (2017)

\section{Submit your manuscript to a SpringerOpen ${ }^{\circ}$} journal and benefit from:

- Convenient online submission

- Rigorous peer review

- Open access: articles freely available online

- High visibility within the field

- Retaining the copyright to your article

Submit your next manuscript at $\gg$ springeropen.com 